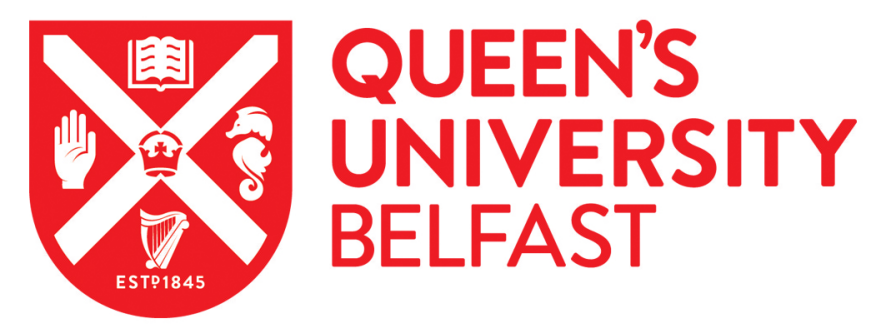

\title{
Singularities in structured meshes and cross-fields
}

Fogg, H. J., Sun, L., Makem, J. E., Armstrong, C. G., \& Robinson, T. T. (2018). Singularities in structured meshes and cross-fields. Computer-Aided Design, 105, 11-25. https://doi.org/10.1016/j.cad.2018.06.002

\author{
Published in: \\ Computer-Aided Design
}

Document Version:

Peer reviewed version

Queen's University Belfast - Research Portal:

Link to publication record in Queen's University Belfast Research Portal

\section{Publisher rights}

Copyright 2018 Elsevier.

This manuscript is distributed under a Creative Commons Attribution-NonCommercial-NoDerivs License

(https://creativecommons.org/licenses/by-nc-nd/4.0/), which permits distribution and reproduction for non-commercial purposes, provided the author and source are cited.

\section{General rights}

Copyright for the publications made accessible via the Queen's University Belfast Research Portal is retained by the author(s) and / or other copyright owners and it is a condition of accessing these publications that users recognise and abide by the legal requirements associated with these rights.

\section{Take down policy}

The Research Portal is Queen's institutional repository that provides access to Queen's research output. Every effort has been made to ensure that content in the Research Portal does not infringe any person's rights, or applicable UK laws. If you discover content in the Research Portal that you believe breaches copyright or violates any law, please contact openaccess@qub.ac.uk. 


\title{
Singularities in structured meshes and cross-fields
}

\author{
Harold J. Fogg ${ }^{\mathrm{a}}$, Liang Sun ${ }^{\mathrm{b}}$, Jonathan E. Makem ${ }^{\mathrm{a}}$, Cecil G. Armstrong ${ }^{\mathrm{b}}$, \\ Trevor T. Robinson ${ }^{\mathrm{b}}$ \\ ${ }^{a}$ Meshing 83 Abstraction, Digital Factory, Simulation and Test Solutions Siemens PLM \\ Software, SIEMENS, Francis House, 112 Hills Road, Cambridge, UK. CB2 1PH \\ ${ }^{b}$ School of Mechanical and Aerospace Engineering, Queen's University Belfast, UK. BT9 \\ $5 A H$
}

\begin{abstract}
Singularities in structured meshes are vertices that have an irregular valency. The integer irregularity in valency is called the singularity index of the vertex of the mesh. Singularities in cross-fields are closely related which are isolated points where the cross-field vectors are defined in its limit neighbourhood but not at the point itself. For a closed surface the genus determines the minimum number of singularities that are required in a structured mesh or in a cross-field on the surface. Adding boundaries and forcing conformity of the mesh or alignment of the cross-field to them also affects the minimum number of singularities required. In this paper a simple formula is derived from Bunin's Continuum Theory for Unstructured Mesh Generation [1] that specifies the net sum of singularity indices that must occur in a cross-field with even numbers of vectors on a face or surface region with alignment conditions. The formula also applies to mesh singularities in quadrilateral and triangle meshes and the correspondence to 3-D hexahedral meshes is related. Some potential applications are discussed.
\end{abstract}

Keywords: structured mesh, cross-field, singularities

\section{Introduction}

Structured grids or quadrilateral (quad) meshes with tensor product structure offer numerical advantages over unstructured meshes. However, when it comes to closed surfaces some disruption of the regular grid structure can be necessary. For instance, in a map projection of a globe, singularities in the longitude-latitude grid occur at the poles. When a surface has boundaries 
forcing the grid to conform to them can also necessitate the introduction of singularities, not just to reduce the distortion of the grid, but there will be a certain minimum number that are essential for facilitating a quad mesh. Although triangle (tri) meshes have a more flexible connectivity than quad meshes, they also have a natural topological structure which must be disrupted for the same reasons.

Cross-fields or N-way rotational symmetry (N-RoSy) vector fields are like vector fields but instead of a single vector being defined at a point they have a set of $N$ equally spread vectors defined. They can be used to describe the 'directionality' of a mesh and their possible singularity combinations are subject to the same conditions as for meshes.

How to determine the requisite cross-field and mesh singularities based on the topological shape of the surface region and the geometric shapes of its boundaries will be covered in this paper.

\subsection{Related work}

A formula was proposed by White et al. [2] for determining if a face is submappable, which means that a logical representation can be found where all edges of the face are horizontal or vertical. First vertices are assigned types, which essentially decides the number of $\pi / 2$ turns (signed with respect to an anticlockwise traversal) between the adjacent edges in a local logical representation. Values of 1, 0, -1 and -2 are assigned to end, side, corner and reversal vertices (cf. Fig. 3). If the sum of the vertex classification values is four it means that the local logical representations of the vertices are consistent with a global logical representation for the face and thus the face is submappable. The check works on the principle that the sum of exterior angles at vertices of a planar polygon must equal $2 \pi$.

The formula was reformulated and generalised to multiply connected faces by Ruiz Girones et al. [3]. For the face to be submappable it must have assigned vertex types such that

$$
\text { \#End- \#Corner }-2 \text { \#Reversal }=4(1-\# \text { Holes })
$$

where \#End, \#Corner and \#Reversal are the number of end, corner and reversal vertex types and \#Holes is the number of inner boundary loops.

Beaufort et al. [4] have recently presented a general formula for determining the numbers of and indices of singularities in tri and quad meshes in consideration of the face's topological shape and boundary alignment constraints. Although it is almost equivalent to that shown here, it is derived by 
a different method of analysing the Euler Formula and topological identities of the meshes and it gives a different perspective of the problem.

Parametrisation-based mesh generation techniques have been intensely developed recently $[5,6]$. Their approach is to initialise and optimise a cross -field to improve its smoothness and in the process establish the number, indices and placements of mesh singularities. A secondary optimisation method solves for a parametrisation and hence mesh that fits with the cross-field. Palacios et al.[7], Ray et al. [8] and Knöppel et al. [9] prove the PoincaréHopf Theorem for cross-fields on smooth and discrete closed oriented surfaces with empty boundaries which relates the net sum of singularity indices (defined slightly differently to here) to its Euler Characteristic.

\subsection{Contributions}

A simple formula is presented which gives the necessary sum of singularity indices in a cross-field with an even number of vectors. It also applies directly to singularities in quad and tri meshes. The formula is equally simple but is more descriptive to that of Ruiz Girones et al. as it determines the net sum of mesh singularity indices that are required for a face, not just whether the face is submappable, and it accounts for more general face topologies. The formula extends the Poincaré-Hopf Theorem for cross-fields to faces with nonempty boundaries on which alignment constraints are enforced. The formula is shown to be sufficient for cross-fields and necessary but not sufficient for quad and tri meshes. For 3-D hex meshes a new condition to verify line singularity networks is derived.

\section{Predicating theories}

\subsection{Euler Characteristic}

If the face $R$ is a regular (i.e. compact orientable) region of surface $S$ its Euler Characteristic can be defined by

$$
\chi(R)=\# V-\# E+\# F,
$$

where $\# V$, \#E, \#F are the number of vertices, edges and facets of a triangulation (or a polygon mesh) on the face. The value does not depend on triangulation, hence it characterises the face [10, Prop. 1, Chp. 4.5]. The Euler Characteristic of a simple face $R=$ disc can be ascertained by analysing a single triangle:

$$
\# V=3, \# E=3, \# F=1 \rightarrow \chi(\text { disc })=1 .
$$


The relationship between the genus, $g(S)$, and Euler Characteristic, $\chi(S)$, of a closed orientable surface with empty boundary $S$ is

$$
g(S)=\frac{2-\chi(S)}{2}
$$

[10, Prop. 4, Chp. 4.5]. The table to the right gives the genera and Euler Characteristics of familiar closed surfaces.

\begin{tabular}{c|c|c}
$S$ & $g(S)$ & $\chi(S)$ \\
\hline sphere & 0 & 2 \\
torus & 1 & 0 \\
double torus & 2 & -2 \\
$\vdots$ & $\vdots$ & $\vdots$
\end{tabular}

\subsection{Global Gauss-Bonnet Theorem}

The Global Gauss-Bonnet Theorem [10, Chp. 4.5] is

$$
\sum_{i=1}^{\# C} \int_{C_{i}} \kappa_{g}(S) d s+\iint_{R} K(S) d A+\sum_{j=1}^{\# C} \gamma_{j}=2 \pi \chi(R)
$$

where $C_{1} \ldots C_{\# C}$ are smooth regular curves embedded on the surface $S$ that together form the boundary $\partial R$ of the regular region $R$. Each $C_{i}$ is positively oriented, i.e. the cross-product of the surface normal with a positive-sensetangent $(\mathbf{n} \times \mathbf{t})$ points into the region. $\gamma_{1} \ldots \gamma_{\# C}$ are the exterior angles at boundary vertices between the pairs of adjacent curves of $C_{1} \ldots C_{\# C}$. The internal angles $\theta_{j}$ are related to the exterior angles $\gamma_{j}$ by

$$
\theta_{j}=\pi-\gamma_{j}
$$

$\kappa_{g}$ denotes the geodesic curvature of the curve $C_{i}$ and $K$ denotes the Gaussian curvature of the surface.

One insight to be made from Eqn. (5) is that by adding in an extra boundary closed loop made from a small circle whose radius $\rightarrow 0$, the LHS goes down by $2 \pi$ and therefore the Euler Characteristic on the RHS must drop by 1 . So, for a face $R$ of a closed orientable surface $S$ with genus $g(S)$ where the boundaries $\partial R$ compose $\# b$ loops that exclude homeomorphic disc regions, the Euler Characteristic of the face is

$$
\begin{aligned}
\chi(R) & =\chi(S)-\# b, \\
& =2-2 g(S)-\# b .
\end{aligned}
$$

For example, a cylinder can be considered topologically to be a region of a sphere between two boundary loops, therefore,

$$
\chi(\text { cylinder })=2-2=0 .
$$




\subsection{A Continuum Theory for Unstructured Mesh Generation in Two Di- mensions}

Bunin [1] explains a precise 'continuum theory' of cross-fields and infinitesimal isotropic quad and tri meshes. Amongst other things, it explains the dependency of the number and indices of singularities that must occur on a face due to its intrinsic shape and boundary alignment constraints. Illustrations of the concepts are given in Fig. 1 and their definitions are given in the following sections.

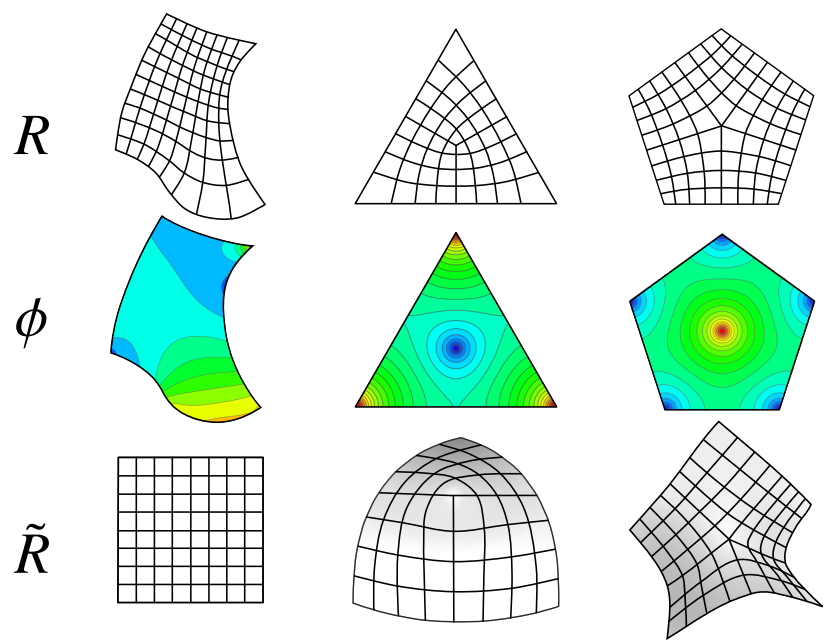

Figure 1: Illustrations of Bunin's continuum theory for quad meshes on three simple faces.

\subsubsection{Definition}

The continuum theory is derived in terms of a conformal mapping of a region (or face), $R$, of a surface $S$ to a region, $\tilde{R}$, of a surface $\tilde{S}$. The surface $\tilde{S}$ is locally flat everywhere except at some isolate points referred to as cone vertices, $\tilde{P}_{\text {cone }}$. The region has boundaries, $\partial R$, composed of curves, $C_{i}$, that join at vertices in $P_{\text {vertex }}$. The continuities of the entities are:

- $S$ is of class $C^{\infty}$,

- $\tilde{S} \backslash \tilde{P}_{\text {cone }}$ is of class $C^{\infty}$,

- $C_{i}$ are of class $C^{l} \mid l \geq 2$.

The conformal mapping is encapsulated by a scalar function, $\phi$, over $S . \tilde{S}$ has a family of geodesics (straight lines) defined to describe the mesh and 
for boundary conformity to be satisfied the family of geodesics encompasses the images of $\partial R$ on $\tilde{S}, \partial \tilde{R}$. This can be defined formally as a $\phi$-manifold which is a Riemannian manifold with metric $\tilde{g}_{u v}$, defined on $R$ with induced metric $g_{u v}$ in $\mathbb{R}^{3}$, less a discrete set of points, $P_{\text {cone, }}$, such that:

1. $g_{u v}$ is conformally related to $\tilde{g}_{u v}$, i.e. $g_{u v}=e^{2 \phi} \tilde{g}_{u v}$, where $\phi \in \mathbb{R}$ on $R \backslash P_{\text {cone }}$.

2. The $\phi$-manifold is locally flat, i.e. its Gaussian curvature $\tilde{K}=0$, on $R \backslash P_{\text {cone }}$.

3. $\phi$ is a real scalar function with continuity $C^{\infty}$ on $R \backslash\left(\partial R \cup P_{\text {cone }}\right)$ and $C^{l-1}$ on $\left(\partial R \backslash P_{\text {cone }}\right)$.

4. A cross-field exists with streamlines that are geodesics of $\tilde{g}_{u v}$ (i.e. straight lines). Its continuity is $C^{\infty}$ on $R \backslash\left(\partial R \cup P_{\text {cone }}\right)$ and $C^{l}$ on $\partial R \backslash P_{\text {cone. }}$.

$P_{\text {cone }}$ corresponds to the inverse images of cone vertices of $\tilde{g}_{u v}, \tilde{P}_{\text {cone }}$, and they can be divided into two independent subsets,

$$
P_{\text {cone }}=P_{k} \cup P_{\text {vertex }} \mid P_{k} \cap P_{\text {vertex }}=\emptyset \text {, }
$$

where $P_{k}$ are the singularity points on the face and $P_{\text {vertex }}$ are the boundary vertices.

A cross-field or N-RoSy field $[7,6]$ has $N$ vectors defined at any point where their magnitudes are irrelevant and the angle between any pair of vectors is a multiple of $2 \pi / N$. Since the cross-field is required to have streamlines $N$ must be even. If $N=4$ the cross-field streamlines plot the edges of an orthogonal quad mesh and if $N=6$ they plot the edges of an equilateral tri mesh, as shown in Fig. 2. Choices for $N>6$ are possible and the $\phi$-manifold
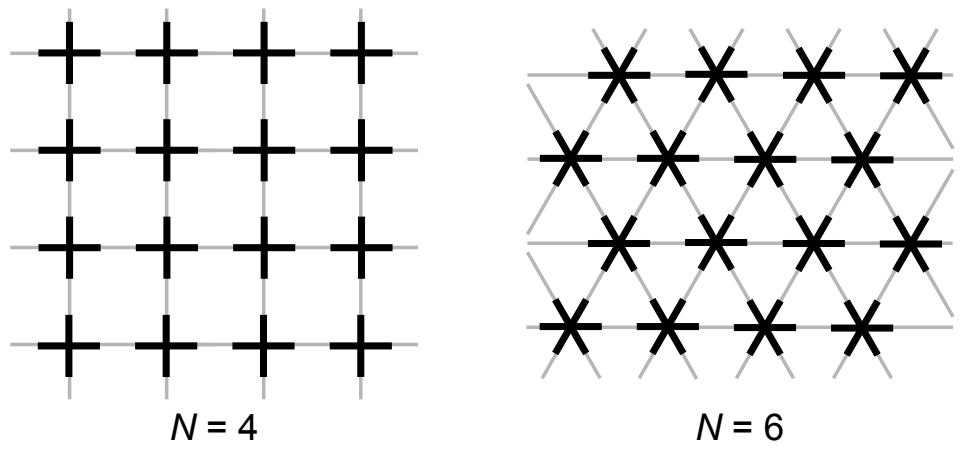

Figure 2: Cross-fields with straight streamlines. 
will describe the behaviour of a cross-field in the same way as for $N=4$ or 6 , however they do not correspond to meshes as obviously.

If cross-field streamlines are spaced at regular intervals on $\tilde{S}$ they describe a reference unit mesh on $\tilde{S}$ and, due to the conformal relation, their inverse images on $S$ describe a locally isotropic mesh. The smaller the interval is set the more the elements approach squares or equilateral triangles on $S$. $\phi$ controls the isotropic stretching of the mapping. The local refinement (or reciprocal of the element size) is

$$
\text { refinement } \propto e^{\phi},
$$

where the proportionality constant is a single number for the entire mesh. Curvatures of cross-field streamlines are related to variation in $\phi$ by

$$
\kappa_{g}=\left\langle\nabla_{S} \phi, \mathbf{e}\right\rangle,
$$

where $\kappa_{g}$ denotes the geodesic curvature of the streamline and its intrinsic normal, $\mathbf{e}$, is defined by $\mathbf{e}=\mathbf{n} \times \mathbf{t}$ where $\mathbf{n}$ and $\mathbf{t}$ are the surface normal and the tangent vector respectively.

\subsubsection{Conditions}

There are four conditions that $\phi$ must satisfy to constitute a valid continuum description.

Condition 1. $\phi$ satisfies the Poisson equation over the face,

$$
\Delta_{S} \phi=K+\sum_{i=1}^{\# P_{k}} k_{i} \frac{2 \pi}{N} \delta_{\mathbf{p}_{i}} \mid k_{i} \in \mathbb{Z} \geq-N .
$$

On the RHS there are two source terms: a distributed source for the Gaussian curvature and point source terms at singularities, $\mathbf{p}_{i} \in P_{k}$, of the crossfield/mesh with strengths of $k_{i} 2 \pi / N$, where the integer $k_{i}$ is the singularity index. The point sources correspond to cone vertices of $\tilde{g}_{u v}$ with angle deficits of $k_{i} 2 \pi / N$.

The singularity index in a mesh relates to the integer irregularity in valency of the vertex at that point. In a cross-field, the singularity index can be determined by taking any closed arc-length parametrised curve, $\alpha(s)$, with continuity $C^{x} \mid x \geq 2$, on the surface that encloses a simple region containing 
the singularity and no others, and evaluating

$$
k=-N\left(1+\frac{1}{2 \pi} \oint_{\alpha} d \theta(s)\right) .
$$

$d \theta(s)$ is the variation of the angle between a cross-field vector and the tangent of $\alpha$ and it has the identity

$$
d \theta(s)=\left(\kappa_{c}-\kappa_{g}\right) d s
$$

where $\kappa_{g}$ is the geodesic curvature of $\alpha$ and $\kappa_{c}$ is the curvature of the crossfield in the tangent direction of $\alpha$, defined as

$$
\kappa_{c}=\left\langle\frac{\partial \mathbf{c}}{\partial s}, \mathbf{c}^{\perp}\right\rangle
$$

where $\mathbf{c}$ is one of the unit cross-field vectors and $\mathbf{c}^{\perp}=\mathbf{n} \times \mathbf{c}$ is an orthogonal unit vector. Note that other authors use a slightly different definition of singularity index, e.g [7], [8] [9]. Fig. 6 provides an intuitive illustration of singularities.

Condition 2. It follows from Eqn. (12) that for boundary alignment to be satisfied the flux across a boundary edge, $C_{i}$, must be

$$
\left.\kappa_{g}\right|_{C_{i}}=\left\langle\nabla_{S} \phi, \mathbf{e}_{i}\right\rangle
$$

where $\mathbf{e}_{i}$ is the intrinsic normal of the curve $C_{i}$.

Condition 3. At boundary vertices (in $P_{\text {vertex }}$ ) where the adjacent boundary curves are tangent discontinuous point sources necessarily occur. Their strength must satisfy

$$
Q=\frac{2 \pi}{\theta_{c}}\left(n_{c} \frac{2 \pi}{N}-\theta_{c}\right),
$$

which involves the corner angle, $\theta_{c}$, and the predetermined vertex classification given by the integer $n_{c}$. It specifies the number of $2 \pi / N$ rotations to match cross-field vectors aligned with the boundary curves either side of the vertex. Or more intuitively, $n_{c}$ specifies the number of adjacent elements to the vertex, as shown in Fig. 3 for quad meshes. The optimum vertex classification that reduces the mesh distortion in the vicinity can be determined by minimising the source strength. 


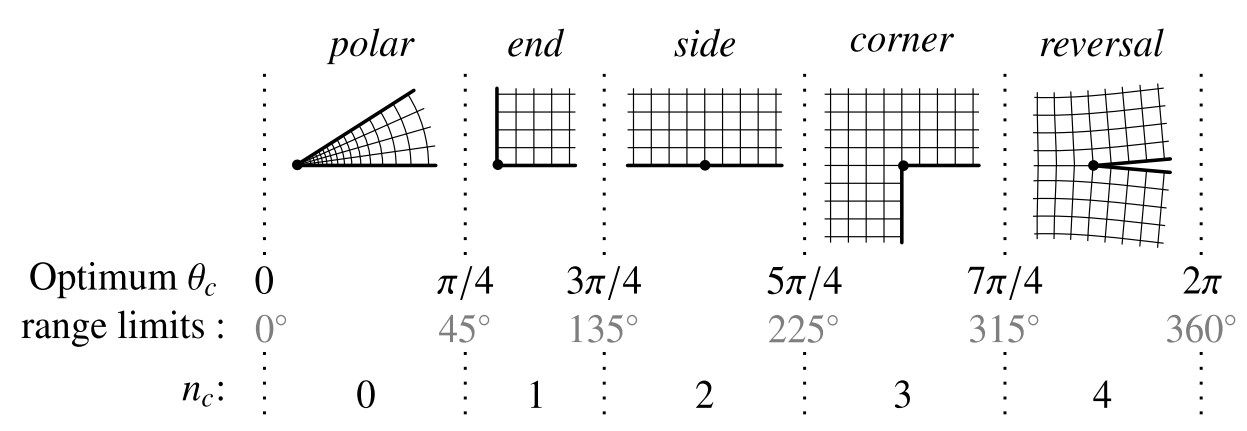

Figure 3: Vertex classification $(N=4)$.

Condition 4. Geodesics of $\tilde{g}_{u v}$ are described by the streamlines of a crossfield. It follows from Eqn. (12) that variation in the cross-field in one direction is controlled by flux in $\phi$ in a perpendicular direction. The condition is that for any $C^{1}$ curve on $S, \alpha$, between two points $a$ and $b$, the total flux through the curve must induce a total turning angle of a cross that is multiple of $2 \pi / N$

$$
\Phi_{\alpha}=\int_{\alpha}\left\langle\nabla_{S} \phi, \mathbf{e}\right\rangle d s=\int_{\alpha} \kappa_{g} d s+\theta_{b}-\theta_{a}+n \frac{2 \pi}{N}, \quad n \in \mathbb{Z},
$$

where $\theta_{a}$ and $\theta_{b}$ are the angles (measured anticlockwise with respect to the local surface normal) between the initial and final curve tangents and one of the $N$ cross-field vectors at those locations at points $a$ and $b$, as shown in Fig. 4 (left).
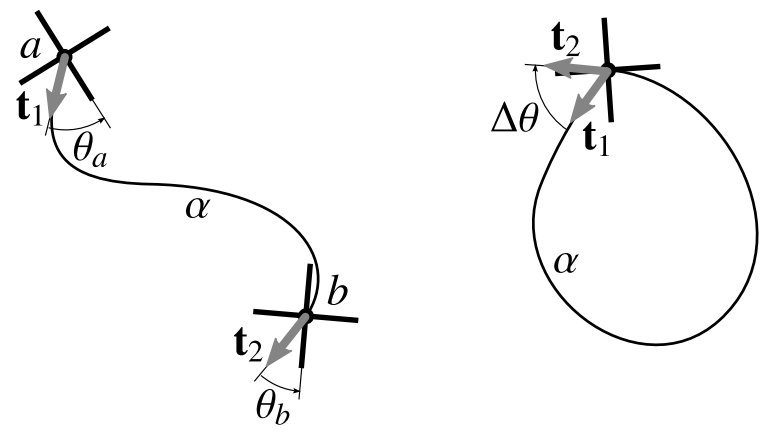

Figure 4: Condition $4(N=4)$. 
For a closed curve the condition is

$$
\Phi_{\alpha}=\oint_{\alpha}\left\langle\nabla_{S} \phi, \mathbf{e}\right\rangle d s=\oint_{\alpha} \kappa_{g} d s-\Delta \theta+n \frac{2 \pi}{N}, \quad n \in \mathbb{Z},
$$

where $\Delta \theta$ is the difference in angle of the initial and final curve tangents, as shown in Fig. 4 (right).

\subsubsection{Solving the continuum description}

Conditions 1-3 (Eqns. (13)-(18)) define a boundary value Poisson equation problem with Neumann boundary conditions (BCs) and distributed and point source loadings. An approximation of the exact solution of $\phi$, which is smooth on $R \backslash P_{\text {cone }}$ and unique up to an additive constant, can be found numerically by solving a linear system in a finite element analysis. For example, the $\phi$ contour plots in Fig. 1 were produced from a thermal solution to a FEA heat conduction analysis. But to do this the number of singularities, their indices and their positions need to be established first.

\subsubsection{Examples of singularities}

According to Eqn. (13), for an isolated singularity at the origin of a polar coordinate system of a planar domain, $\phi$ is given by

$$
\phi(r)=\frac{k}{N} \ln r+\text { const. }
$$

Examples of surface plots of $\phi$ around singularities are shown in Fig. 5. Ex-

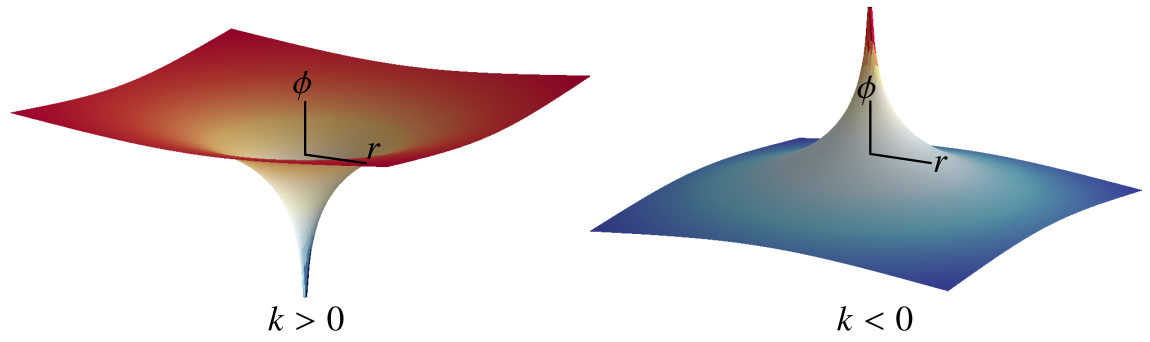

Figure 5: Surface plots of $\phi$ around singularities.

amples of the corresponding locally isotropic quad and tri meshes for different singularity indices are shown in Fig. 6 . These were produced by numerically tracing streamlines according to Eqns. (12) and (21) and spaced with a refinement according to Eqn. (11). Isotropic hexagon meshes around singularities 
are also shown. Since regular hexagons can be formed by joining six adjacent equilateral triangles the hexagon mesh examples for $k=0,1,-1$ are produced from the tri mesh examples of $k=0, k=2, k=-2$.

\section{Formula for singularities in cross-fields and meshes from Bunin's Continuum Theory}

(1) For a boundary conforming cross-field on a face $R$ with Euler characteristic $\chi(R)$ and prescribed vertex types $n_{c 1} \ldots n_{c \# V}$ for the start vertices of boundary curves $C_{1}, \ldots C_{\# C}$, the net sum of the indices of singularities, $P_{k}$, must satisfy

$$
\sum_{i=1}^{\# P_{k}} k_{i}=-N \chi(R)+\sum_{j=1}^{\# V}\left(\frac{N}{2}-n_{c_{j}}\right) .
$$

(2) The singularities in quad or tri meshes must satisfy Eqn. (22) with $N=$ $4, N=6$ respectively.

Proof. This can be proven straightforwardly by setting up a necessary condition from Bunin's Continuum Theory and simplifying it using the GaussBonnet Theorem.

\subsection{Consistency flux balance condition}

The Poisson equation describes steady-state fields, therefore over any subregion of the face the total flux of $\phi$ must balance in order for a solution to exist that satisfies Eqn. (13) in Condition 1. Assessing the whole face the flux balance condition is

$$
\Phi_{\partial R}+\Phi_{R}+\Phi_{k}=0 .
$$

The total flux into the face through boundaries from curves and vertices is

$$
\Phi_{\partial R}=\sum_{i=1}^{\# C} \int_{C_{i}} \kappa_{g} d s+\sum_{j=1}^{\# V}\left(\frac{2 \pi}{N} n_{c_{j}}-\theta_{j}\right),
$$

where $\# C$ and $\# V$ are the the numbers of curves and vertices and they must

equal. The total flux from the Gaussian curvature of the surface over the face is

$$
\Phi_{R}=\iint_{R} K d A
$$



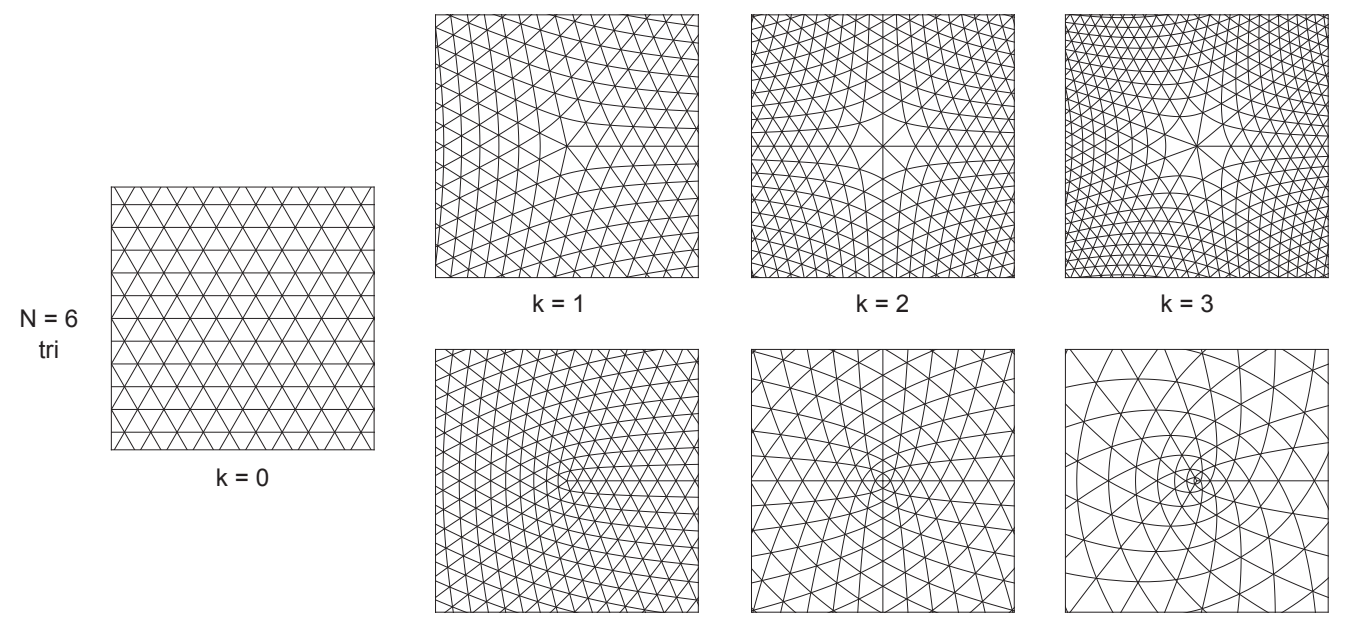

$\mathrm{k}=2$
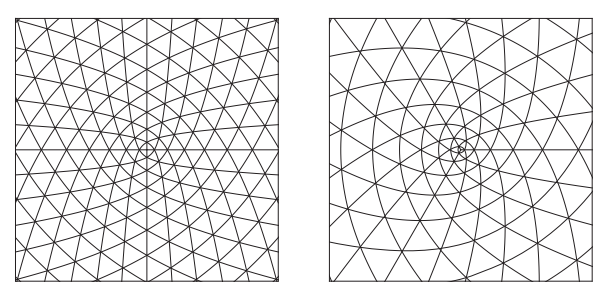

$k=-2$

$\mathrm{k}=-3$
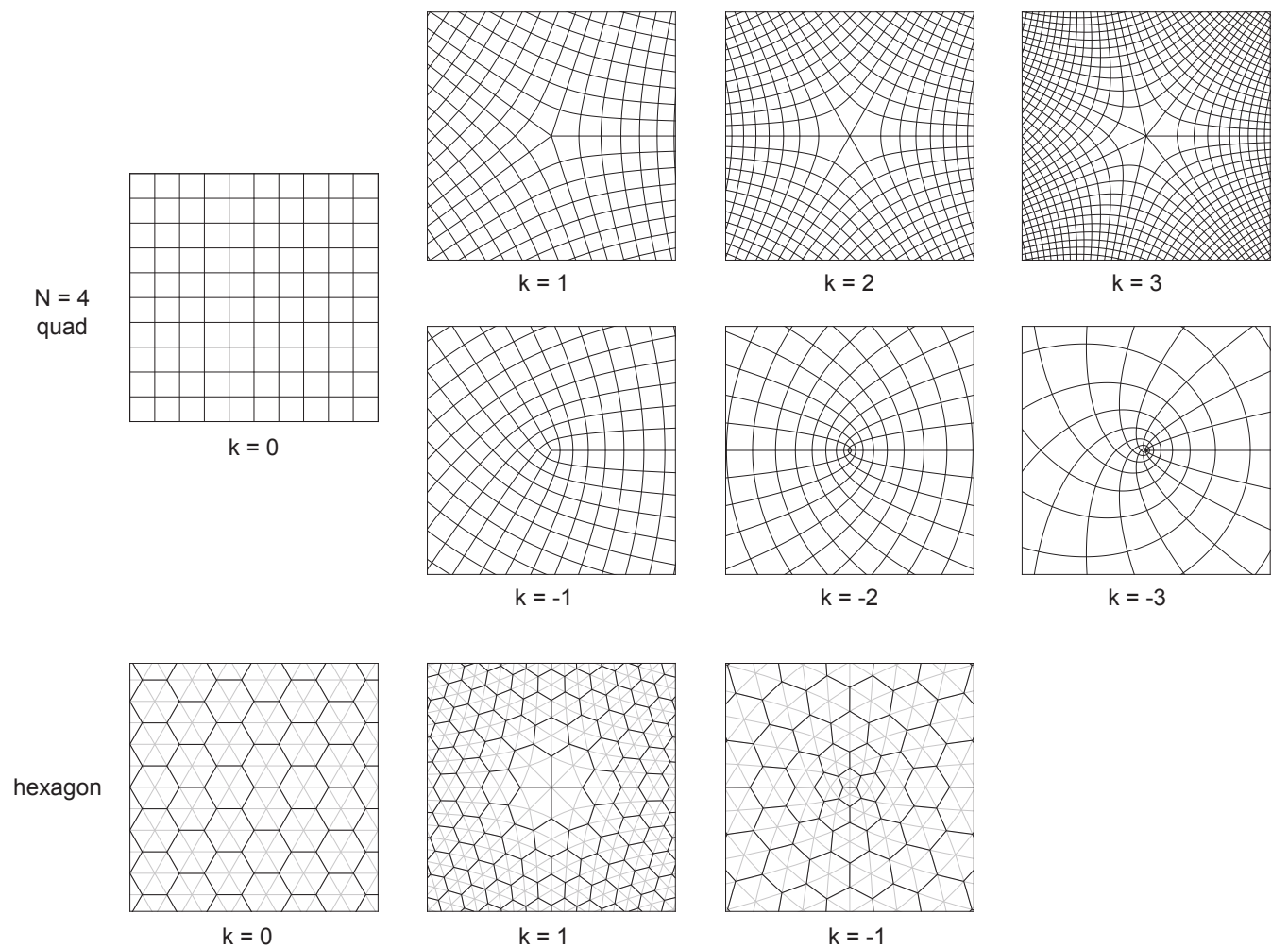

Figure 6: Examples of isotropic tri $(N=6)$, quad $(N=4)$ and hexagon meshes around singularities with indices $k$. 
and the total flux from the mesh singularities is

$$
\Phi_{k}=\sum_{i=1}^{\# P_{k}} k_{i} \frac{2 \pi}{N}
$$

The consistency flux balance condition requires that

$$
\Phi_{\partial R}+\Phi_{R}+\Phi_{k}=0
$$

Equations (24) and (25) can be combined and simplified as follows:

$$
\begin{aligned}
& \Phi_{\partial R}+\Phi_{R}= \sum_{i=1}^{\# C} \int_{C_{i}} \kappa_{g} d s+\iint_{R} K d A+\sum_{j=1}^{\# V}\left(\frac{2 \pi}{N} n_{c_{j}}-\theta_{j}\right) \\
&= 2 \pi \chi(R)-\sum_{j=1}^{\# C} \gamma_{j}+\sum_{j=1}^{\# V}\left(\frac{2 \pi}{N} n_{c_{j}}-\theta_{j}\right) \\
& \ldots \text { Gauss-Bonnet (Eqn. (5)) } \\
&=2 \pi \chi(R)-\sum_{j=1}^{\# V}\left(\pi-\theta_{j}\right)+\sum_{j=1}^{\# V}\left(\frac{2 \pi}{N} n_{c_{j}}-\theta_{j}\right) \\
& \quad \ldots \text { Eqn. }(6) \text { and } \# V=\# C \\
&=2 \pi \chi(R)-\sum_{j=1}^{\# V}\left(\pi-\frac{2 \pi}{N} n_{c_{j}}\right) .
\end{aligned}
$$

Then rearranging Eqn. (27) and substituting we get

$$
\begin{gathered}
-\Phi_{k}=\Phi_{\partial R}+\Phi_{R} \\
-\sum_{i=1}^{\# P_{k}} k_{i} \frac{2 \pi}{N}=2 \pi \chi(R)-\sum_{j=1}^{\# V}\left(\pi-\frac{2 \pi}{N} n_{c_{j}}\right) \\
\sum_{i=1}^{\# P_{k}} k_{i}=-N \chi(R)+\sum_{j=1}^{\# V}\left(\frac{N}{2}-n_{c_{j}}\right) \cdot \square
\end{gathered}
$$

Thus, only if the condition on the singularities is satisfied can the consistency flux balance condition be satisfied, hence a $\phi$-manifold be established. With $\phi$ (or even just $\nabla_{S} \phi$ by Eqn. (12)) a cross-field can be established. 
Not every tri mesh or quad mesh has element angles that are uniformly $\pi / 3$ or $\pi / 2$ respectively, in which case a cross-field cannot be found which describes them exactly. However, a generalisation of cross-fields called NPolyVector fields can describe all tri or quad meshes and Diamanti et al. [11] have shown that the topology and singularities of a N-PolyVector field is equivalent to an associated 'canonical' cross-field. Therefore, conditions that apply to singularities in cross-fields also apply to N-PolyVector fields and to general quad and tri meshes.

\subsection{Examples}

Examples and validations of the formula are given in Fig. 7, 8 and 9. Singularities are marked and boundary vertices which affect the calculation are also marked with a number for $n_{c}$.

In Fig. 7 multiblock decompositions of a selection of sample faces are shown and their singularity index sums are demonstrated to agree with the resultants of the formula. In Fig. 8 two meshes of the same face are shown which were generated by a paving and multiblock decomposition method in Siemens Simcenter 3D [12]. The latter mesh has two vertex classification interpretations depending on whether the two vertices on the boundaries indicated are treated as singularities (VC1) or boundary vertices (VC2). The formula is shown to hold for both. A tri mesh example of the same face is shown in Fig. 9.

\section{Sufficiency of the formula}

\subsection{Satisfying condition 4}

Any combination of singularities that result in a balanced total flux gives a well-posed Poisson equation problem with Neumann BCs that can be solved for a unique $\phi$ up to an additive constant, or equivalently a unique $\nabla_{S} \phi[13]$. However, not all solutions satisfy condition 4 .

\subsubsection{Simply connected faces}

On simply connected faces (i.e. topological discs and spheres) the $\nabla_{S} \phi$ solution satisfying conditions 1-3 automatically satisfies condition 4.

Proof. For a topological disc, $R$, with a single boundary loop composed of smooth boundary curves, $C_{i}$ consider a simple subregion, $\breve{R}$, as illustrated in Fig. 10. The boundary is composed of $C_{1}, \ldots C_{\# C}$ where the start and end 


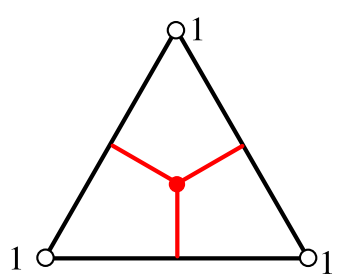

$\chi=1$

$\sum\left(2-n_{c_{j}}\right)=3(2-1)=3$

$\Rightarrow \sum k_{i}=-4(1)+3=-1$

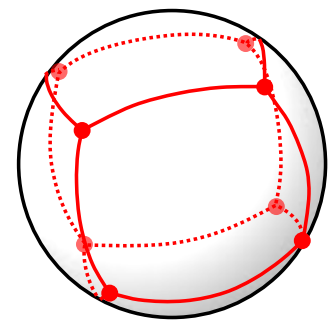

$$
x=2
$$

$\sum\left(2-n_{c_{j}}\right)=0$
$\Rightarrow \sum k_{i}=-4(2)+0=-8$

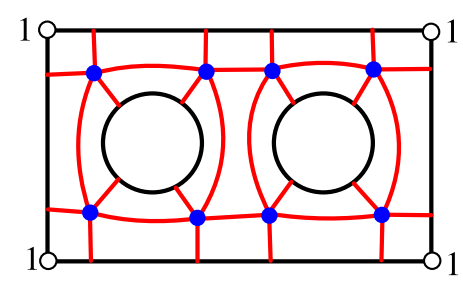

$\chi=1-2=-1$

$\sum\left(2-n_{c_{j}}\right)=4(2-1)=4$

$\Rightarrow \sum k_{i}=-4(-1)+4=8$

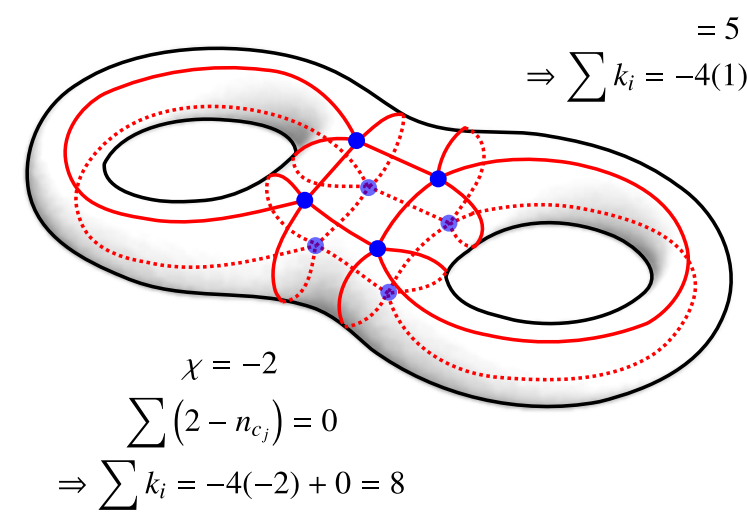

Figure 7: Some quad $(N=4)$ multiblock decompositions of faces and validation calculations of the formula.

curves $C_{1}$ and $C_{\# C}$ are truncated by the points $a$ and $b$, and these are joined by a non self-intersecting smooth curve $\alpha$. The corner angles are labelled $\theta_{c_{1}} \ldots \theta_{c_{\# C-1}}$ and $\theta_{c_{a}}, \theta_{c_{b}}$. The Gauss Bonnet Theorem for the subregion is

$$
\sum_{i=1}^{\# C} \int_{C_{i}} \kappa_{g} d s+\int_{\alpha} \kappa_{g} d s+\iint_{\breve{R}} K d A+\sum_{i=1}^{\# C-1}\left(\pi-\theta_{c i}\right)+\left(\pi-\theta_{c a}\right)+\left(\pi-\theta_{c b}\right)=2 \pi
$$

The flux balance condition on $\phi$ is

$$
\Phi_{\partial \breve{R}}+\Phi_{\breve{R}}+\Phi_{k}=0 .
$$

The total flux over the subregion is

$$
\Phi_{\breve{R}}=\iint_{\breve{R}} K d A .
$$



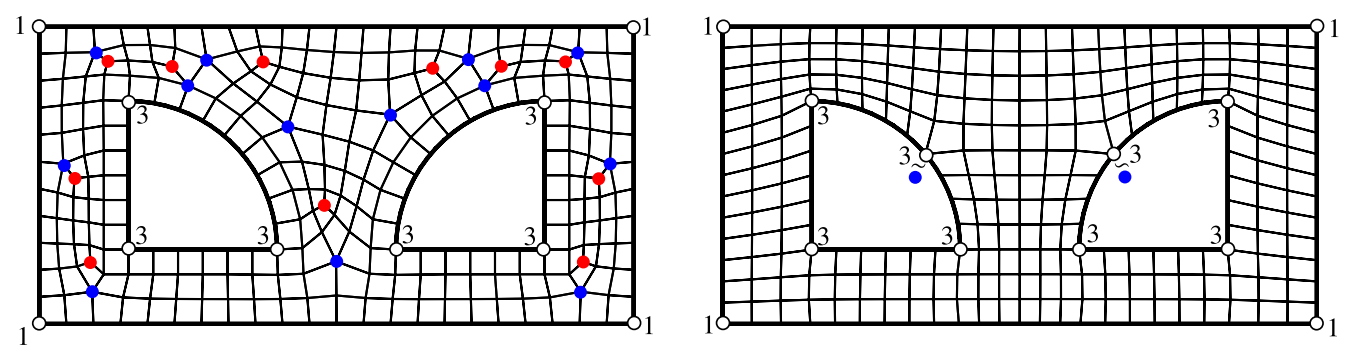

Prediction (VC1):

$\chi=-1$

$N=4$

Prediction (VC2):

$\sum\left(2-n_{c_{j}}\right)=4(2-1)+6(2-3)=-2$

$\Rightarrow \sum k_{i}=-4(-1)+(-2)=2$

Inspection:

$\sum k_{i}=11(-1)+13(1)=2(\mathrm{VC} 1) \checkmark$

$\sum\left(2-n_{c_{j}}\right)=4(2-1)+8(2-3)=-4$

$\Rightarrow \sum k_{i}=-4(-1)+(-4)=0$

Inspection:

$\sum k_{i}=2(\mathrm{VC} 1)$ or $0(\mathrm{VC} 2) \checkmark$

Figure 8: A paved quad mesh (left) and multiblock mesh (right) $(N=4)$ generated in Siemens Simcenter 3D [12]. Validiation calculations of the formula are given below.

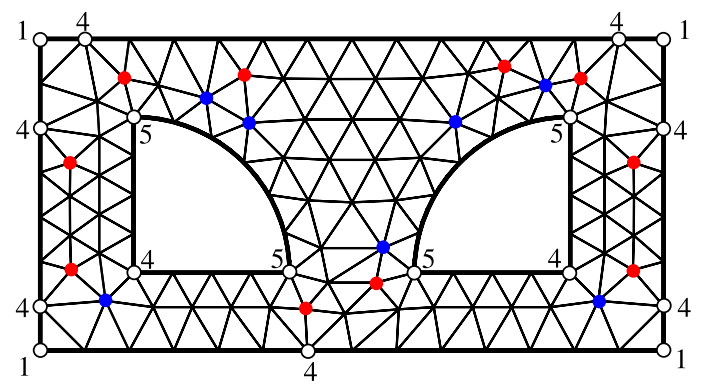

$$
\begin{gathered}
\chi=-1, N=6 \\
\text { Prediction: } \\
\sum\left(3-n_{c_{j}}\right)=4(3-1)+9(3-4)+4(3-5) \\
=-9 \\
\Rightarrow \sum k_{i}=-6(-1)+(-9)=-3
\end{gathered}
$$

Inspection:

$\sum k_{i}=10(-1)+7(1)=-3 \checkmark$

Figure 9: A paved tri mesh $(N=6)$ generated in Siemens Simcenter 3D with validation calculations.

The total flux though the boundaries is

$$
\Phi_{\partial \breve{R}}=\sum_{i=1}^{\# C} \int_{C_{i}} \kappa_{g} d s+\sum_{i=1}^{\# C-1}\left(\frac{2 \pi}{N} n_{c_{i}}-\theta_{i}\right)+\Phi_{\alpha},
$$

where $\Phi_{\alpha}$ is the flux through $\alpha$. The total flux from $\# P_{k}$ mesh singularities in the region is

$$
\Phi_{k}=\sum_{j=1}^{\# P_{k}} k_{j} \frac{2 \pi}{N}
$$




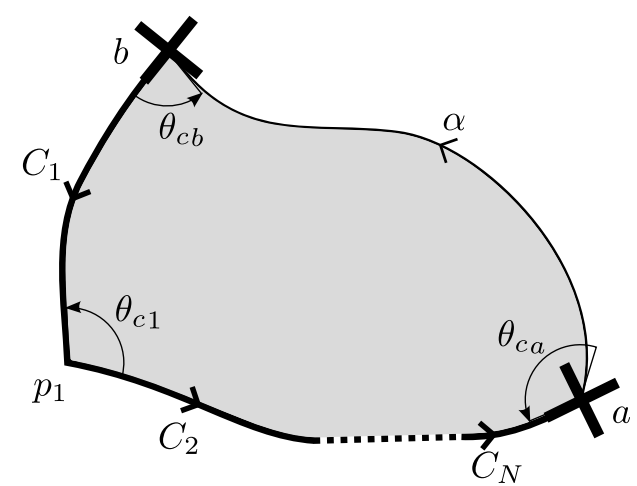

Figure 10: A subregion, $\breve{R}$, of a simply connected face.

Comparing Eqn. (35) and Eqns. (36), (37), (38) and (39)

$$
-\int_{\alpha} \kappa_{g} d s+\Phi_{\alpha}+\sum_{i=1}^{\# C-1}\left(-\pi+\frac{2 \pi}{N} n_{c_{i}}\right)=-\theta_{c a}-\theta_{c b} .
$$

With respect to the definition of Condition $4, \theta_{a}=\theta_{c a}$ and $\theta_{b}=2 \pi / N-\theta_{c b}$. Therefore,

$$
\Phi_{\alpha}=\int_{\alpha} \kappa_{g} d s+\theta_{b}-\theta_{a} \underbrace{-\frac{2 \pi}{N}-\sum_{i=1}^{\# C-1}\left(-\pi+\frac{2 \pi}{N} n_{c_{i}}\right)-\sum_{j=1}^{\# P_{k}} k_{j} \frac{2 \pi}{N}}_{n \frac{2 \pi}{N} \mid}
$$

which proves that the flux through $\alpha$ always satisfies Condition 4 .

The more simple analogous analysis for a topological sphere with empty boundary gives the same result.

\subsubsection{Multiply connected surfaces}

For multiply connected surfaces the total flux to satisfy condition 4 must be enforced between separate boundary closed loops and around surface handles. This can be achieved by creating cuts until a simply connected face is produced where the cuts appear twice with opposite senses in its boundary closed loops and Neumann BCs are applied across the cuts so as to satisfy the required total flux. In a finite element analysis the BCs that minimise the Dirichlet energy, $\int_{R}\left\|\nabla_{S} \phi\right\|^{2} d A$, may be found as part of the solution by using multiple point constraints to enforce the total fluxes across the cuts. However, the $\nabla_{S} \phi$ solution (that is unique for the applied BCs) may not allow for a corresponding continuous $\phi$ to exist. 


\subsection{Integrability}

The behaviour of a cross-field is governed by the surface gradient of $\phi$ by Eqn. (12). Considering the unknown to be $\mathbf{v}:=\nabla_{S} \phi$, Eqn. (13) becomes

$$
\nabla_{S} \cdot \mathbf{v}=K+\sum_{i=1}^{\# P_{k}} k_{i} \frac{2 \pi}{N} \delta_{\mathbf{p}_{i}} \mid k_{i} \in \mathbb{Z} \geq-N
$$

A unique cross-field follows from a continuous $\mathbf{v}$ on $R \backslash P_{\text {cone }}$ once a cross orientation at one point is set. The continuity of $\mathbf{v}$ is $C^{\infty}$ on $R \backslash\left(\partial R \cup P_{\text {cone }}\right)$ and $C^{l-2}$ on $\partial R \backslash P_{\text {cone }}$.

Helmholtz Theorem states that any vector field can be decomposed into the gradient of a scalar potential and the curl of a vector potential. Therefore $\mathbf{v}$ is irrotational from its definition. It follows that $\mathbf{v}$ is locally integrable which means that over any simply connected subregion, $\breve{R}$ :

1. A continuous $\phi$ can be found such that $\mathbf{v}=\nabla_{S} \phi$.

2. The circulation around the simple closed path perimeter is zero,

$$
\oint_{\partial \breve{R}}\langle\mathbf{v}, \mathbf{t}\rangle d s=0 .
$$

If these are true over all (multiply connected or not) subregions then $\mathbf{v}$ is globally integrable. In that case a continuous cross-field, $\phi$ and corresponding isotropic mesh can all be found. But this is not the case in general.

Since $\mathbf{v}$ does not define the directions of cross-field streamlines but instead it defines the maximal rate of change of angle of the cross-field, $\mathbf{v}$ solutions with circulation can have corresponding cross-fields that admit orthogonal (anisotropic) quad meshes so long as cross-field streamlines don't spiral. Spiralling occurs when,

$$
\begin{gathered}
\oint_{\alpha}\langle\mathbf{v}, \mathbf{t}\rangle d s \neq 0 \text { and } \\
\oint_{\alpha}\langle\mathbf{v}, \mathbf{e}\rangle d s=\oint_{\alpha} \kappa_{g} d s-\Delta \theta .
\end{gathered}
$$

That is, when $n=0$ in Eqn. (20) for a closed curve with a non-zero circulation of $\mathbf{v}$.

A simple example is shown in Fig. 11 where a square face has a rotated square hole. Condition 4 forces a total flux to occur between the outer and inner boundary loops. A continuous $\mathbf{v}$ solution can be found satisfying these flux conditions but it is not globally integrable due to the circulation of $\mathbf{v}$ 

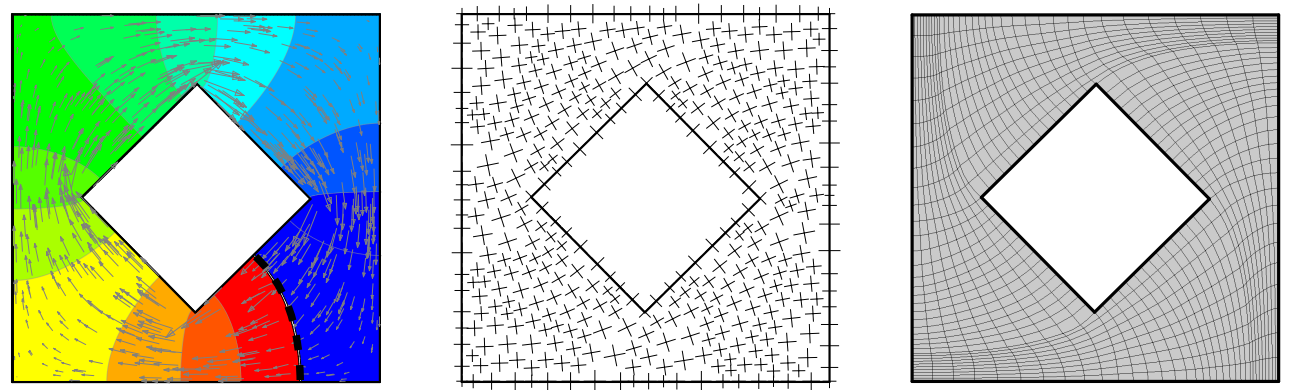

Figure 11: Square face with a rotated square hole. Continuous $\mathbf{v}$ and discontinuous $\phi$ solutions for $N=4$ (left), related boundary-aligned cross-field (centre) and corresponding orthogonal quad mesh (right).

around the hole, and therefore it cannot be integrated to give a continuous $\phi$ solution. The $\phi$ solution shown in Fig. 11 (left) has a discontinuous step change across a cut (dashed line) where condition 4 is imposed. The streamlines of the associated cross-field to $\mathbf{v}$ in Fig. 11 (centre) do not spiral as $n=4$ (plus-minus depending on traversal direction) in Eqn. (20) for a closed path around the hole. Therefore, the cross-field admits an orthogonal (anisotropic) quad mesh as shown in Fig. 11 (right) despite the global non-integrability of $\mathbf{v}$.

A counterpoint example where a cross-field of a continuous globally nonintegrable $\mathbf{v}$ does not admit a mesh is the nautilus face shown in Fig. 12. The circulation of $\mathbf{v}$ for all closed curves around the hole cannot be zero due to Condition 4, which prevents the existence of a continuous $\phi$. The streamlines clearly spiral as indicated which prevents the existence of a corresponding mesh.
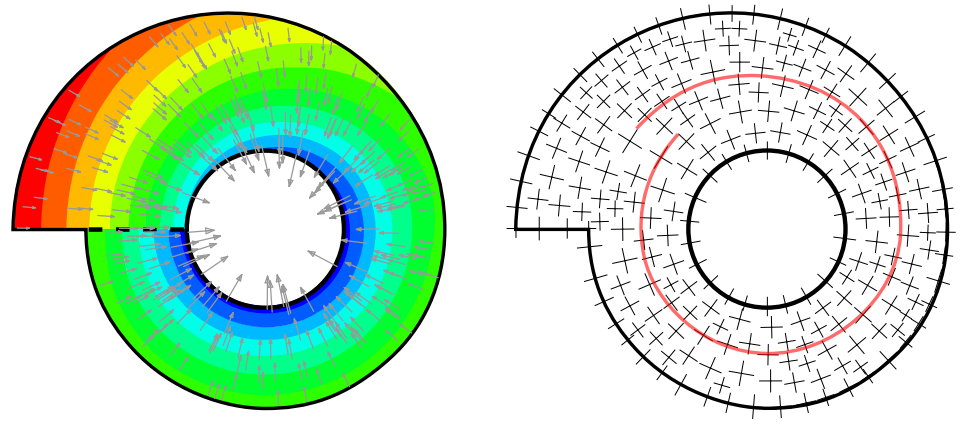

Figure 12: Continuous $\mathbf{v}$, discontinuous $\phi$ (left) and cross-field with spiralling streamline (right) for $N=4$ on nautilus face. 
The disc face in Fig. 13 has continuous $\mathbf{v}, \phi$ and cross-field solutions and thus a corresponding isotropic quad mesh is realisable. Condition 4 allows
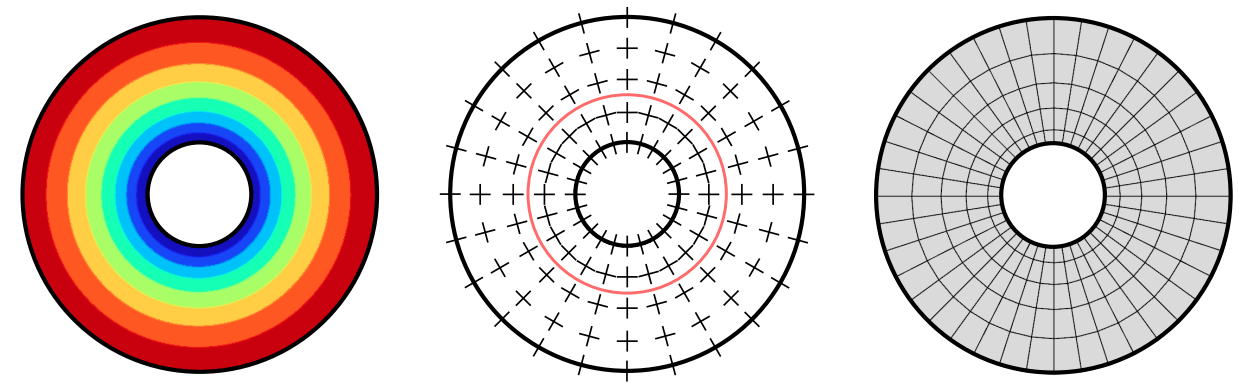

Figure 13: Continuous $\phi$ (left), cross-field with loop streamline (centre) for $N=4$ on on disc face. Corresponding isotropic quad mesh (right).

the circulation around the hole to be zero and therefore despite having $n=0$ in Eqn. (43) for a closed curve around the hole the streamlines do not spiral but close onto themselves.

\subsection{Sufficiency summary}

Any collection of singularities positioned anywhere on a face with indices that satisfy the formula are sufficient for a continuous vector field $\mathbf{v}:=\nabla_{S} \phi$ satisfying Conditions 1-4 to exist. Hence, a boundary aligned cross-field with an even number of vectors (i.e. $N$ is even) exists.

For quad and tri meshes, although cross-fields with $N=4$ and $N=6$ exist they may not describe realisable meshes. If $\mathbf{v}$ is globally integrable then a corresponding continuous $\phi$ exists and a corresponding mesh always exists. Moreover, a corresponding isotropic mesh exists. This is always possible for simply connected faces. If $\mathbf{v}$ is not globally integrable, which can only happen for multiply connected faces, a corresponding continuous $\phi$ does not exists but the cross-field may still be compatible with a quad mesh if spiralling of cross-field streamlines does not occur. These meshes cannot be isotropic however.

So, the formula is necessary and sufficient for cross-fields and necessary but not sufficient for meshes. 


\section{Discussion of related formulae}

\subsection{Relation to Ruiz Girones et al. check}

Equation (22) can be shown to be equivalent to Eqn. (1) for topological discs. Using the variables in (1), the number of corners $\# V$ is

$$
\# V=\# \text { End }+\# \text { Corner }+\# \text { Reversal }
$$

and the total number of elements at the vertices can be expressed as

$$
\sum n_{c_{i}}=\# \text { End }+3 \# \text { Corner }+4 \# \text { Reversal } .
$$

From Eqn. (22)

$$
\begin{aligned}
\sum k_{i}= & -4 \chi+2(\# \text { End }+\# \text { Corner }+\# \text { Reversal })- \\
& (\# \text { End }+3 \# \text { Corner }+4 \# \text { Reversal }) \\
= & \# \text { End }-\# \text { Corner }-2 \# \text { Reversal }-4 \chi .
\end{aligned}
$$

For a topological disc with \#Holes inner boundary loops $(\chi=1-$ \#Holes $)$ and setting $\sum k_{i}=0$, then

$$
\text { \#End- \#Corner }-2 \text { \#Reversal }=4(1-\text { \#Holes }) .
$$

5.2. Formula for singularities in 2-D structured meshes from the Euler Formula

Beaufort et al. [4] have recently developed another method starting from the Euler Formula and considering connectivity relationships for establishing a similar formula for mesh singularities. That method will be discussed here (with some modified terminology) and slightly generalised to cover hexagon as well as quad and tri meshes.

The Euler Formula which defines the Euler Characteristic of a face, $R$, is (Eqn. (2))

$$
\chi(R)=\# V-\# E+\# F .
$$

The edges and vertices can be split into two groups for those on the boundary (subscript $b$ ) and those in the interior (subscript in) respectively,

$$
\begin{gathered}
\# V=\# V_{b}+\# V_{\mathrm{in}}, \\
\# E=\# E_{b}+\# E_{\mathrm{in}}, \\
\# V_{b}=\# E_{b} .
\end{gathered}
$$


If each facet (as an element is called here for clarity of syntax) is a $N_{\text {facet-gon }}$ with $N_{\text {facet }}$ edges (and vertices) the following identity holds

$$
\begin{aligned}
N_{\text {facet }} \# F & =2\left(\# E_{\text {in }}\right)+\# E_{b} \\
& =2 \# E-\# E_{b} \quad \ldots \text { Eqn. }(51) .
\end{aligned}
$$

Substituting for \#E using Eqn. (49) and rearranging yields

$$
2 \# V-\# E_{b}+\left(2-N_{\text {facet }}\right) \# F=2 \chi \text {. }
$$

Another identity is

$$
\sum_{i}^{\# V} \operatorname{valence}\left(v_{i}\right)=N_{\text {facet }} \# F
$$

where valence $\left(v_{i}\right)$ denotes the valence of vertex $v_{i}$. For vertices in the interior let

$$
\operatorname{valence}\left(v_{i}\right)=v_{\text {reg }}+k_{i}
$$

where $v_{\text {reg }}$ is the decided regular valency of the mesh and $k_{i}$ is the index of the singularity at the vertex. For vertices on the boundary

$$
\operatorname{valence}\left(v_{i}\right)=n_{c_{i}}
$$

as per Fig. 3. Then Eqn. (55) can be written as

$$
\# V_{\text {in }} v_{\text {reg }}+\sum_{i}^{\# V_{\text {in }}} k_{i}+\sum_{i}^{\# V_{b}} n_{c_{i}}=N_{\text {facet }} \# F \text {. }
$$

Combining Eqns. (50), (54) and (58) gives a formula for the sum of singularity indices

$$
\sum_{i}^{\# V_{\text {in }}} k_{i}=\frac{2 N_{\text {facet }}}{2-N_{\text {facet }}} \chi-\# V\left(\frac{2 N_{\text {facet }}}{2-N_{\text {facet }}}+v_{\text {reg }}\right)+\sum_{i}^{\# V_{b}}\left(\frac{N_{\text {facet }}}{2-N_{\text {facet }}}+v_{\text {reg }}-n_{c_{i}}\right) .
$$

We want to achieve a formula that is independent of the number of vertices in the mesh so let

$$
v_{\text {reg }}=\frac{-2 N_{\text {facet }}}{2-N_{\text {facet }}}
$$


The only integer solutions for $\left(N_{\text {facet }}, v_{\text {reg }}\right)$ are $(3,6),(4,4)$ and $(6,3)$ (Fig. 14) which correspond to structured tri, quad and hexagon meshes respectively. Thus, these are the only possible polygon structured meshes with defined regular valencies that have topologies that are independent of the number of elements in the mesh. Substituting for $v_{\text {reg }}$ in terms of $N_{\text {facet }}$

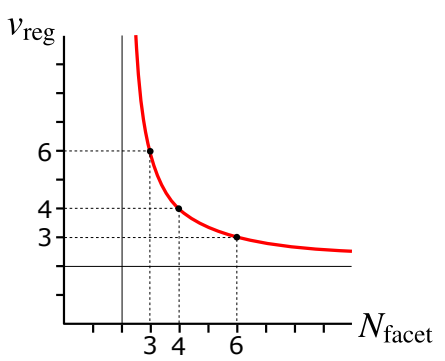

Figure 14: Plot of $v_{\text {reg vs }} N_{\text {facet }}$ gives the formula

$$
\sum_{i}^{\# V_{\text {in }}} k_{i}=\frac{2 N_{\text {facet }}}{2-N_{\text {facet }}} \chi+\sum_{i}^{\# V_{b}}\left(\frac{-N_{\text {facet }}}{2-N_{\text {facet }}}-n_{c_{i}}\right) .
$$

Or substituting for $N_{\text {facet }}$ in terms of $v_{\text {reg }}$ gives the formula

$$
\sum_{i}^{\# V_{\text {in }}} k_{i}=-v_{\mathrm{reg}} \chi+\sum_{i}^{\# V_{b}}\left(\frac{v_{\mathrm{reg}}}{2}-n_{c_{i}}\right)
$$

This is identical to Eqn. (22) except it involves the regular valency of the mesh, $v_{\text {reg }}$, in place of the number of vectors in the cross-field, $N$. Eqn. (22) is derived only for even $N$ cross-fields whereas Eqn. (62) has been shown to apply for $v_{\text {reg }}=3$ (for hexagon meshes) as well as $v_{\text {reg }}=4,6$ (for quad and tri meshes).

\section{Singularities in quad and hexahedral meshes in 3-D bodies}

Quad and hexahedral (hex) mesh singularities that can occur in a 3-D body will be discussed in this section. A body is assumed to mean a closed volume between connected sets of orientable boundary faces called shells (i.e an outer surface boundary and inner void boundaries).

\subsection{Quad mesh singularities on boundary faces of 3-D body}

On a shell of a $3-\mathrm{D}$ body, let $\# V$, $\# E$ and $\# F$ be the total number of vertices, edges and faces. From Eqn. (22) with $N=4$, the sum of quad 
singularity indices on the shell is

$$
\begin{aligned}
\sum_{i=0}^{\left.\# P_{k}\right|_{\text {shell }}} k_{i} & =\sum_{p=1}^{\# F}\left(-4 \chi\left(f_{p}\right)+\sum_{q=1}^{\# V\left(f_{p}\right)}\left(2-n_{c_{q}}\right)\right) \\
& =-4 \sum_{p=1}^{\# F} \chi\left(f_{p}\right)+\sum_{p=1}^{\# F} \sum_{q=1}^{\# V\left(f_{p}\right)}\left(2-n_{c_{p q}}\right)
\end{aligned}
$$

where $\# V\left(f_{p}\right)$ is the number of vertices for face $f_{p}$. Let $\# V_{m}$ be the number of vertices with valency $m$ (the number of faces incident at the vertex), and $\# F_{n}$ be the number of $n$-sided faces.

$$
\begin{aligned}
& \# V=\sum_{m=1}^{\infty} \# V_{m}, \\
& \# F=\sum_{n=0}^{\infty} \# F_{n} .
\end{aligned}
$$

First, consider cases where each face in the body is homomorphic to a closed disc, i.e $\chi\left(f_{p}\right)=1$,

$$
\sum_{i=0}^{\left.\# P_{k}\right|_{\text {shell }}} k_{i}=\sum_{n=1}^{\infty}\left((-4+2 n) \# F_{n}\right)-\sum_{p=1}^{\# F} \sum_{q=1}^{\# V\left(f_{p}\right)} n_{c_{p q}}
$$

The last term can be expressed in terms of vertices,

$$
\sum_{i=0}^{\left.\# P_{k}\right|_{\text {shell }}} k_{i}=\sum_{n=1}^{\infty}\left((-4+2 n) \# F_{n}\right)-\sum_{p=1}^{\# V} \sum_{q=1}^{\# F\left(v_{p}\right)} n_{c_{p q}}
$$

where $\# F\left(v_{p}\right)$ is the number of faces for vertex $v_{p}$. On the other hand, there is a double counting identity

$$
\sum_{m=1}^{\infty} m \# V_{m}=\sum_{n=1}^{\infty} n \# F_{n}=2 E .
$$

So, by the the Euler Formula (Eqn. (2))

$$
\chi(\text { shell })=\# V-\frac{1}{2} \sum_{n=1}^{\infty} n \# F_{n}+\sum_{n=1}^{\infty} \# F_{n} .
$$


Multiplying both sides by -4 gives

$$
-4 \chi(\text { shell })=-4 \# V+\sum_{n=1}^{\infty}\left((-4+2 n) \# F_{n}\right) .
$$

Combining Eqns. (68) and (71) gives

$$
\sum_{i=0}^{\left.\#_{P} k\right|_{\text {shell }}} k_{i}=-4 \chi(\text { shell })+\sum_{p=1}^{\# V}\left(4-\sum_{q=1}^{\# F\left(v_{p}\right)} n_{c_{p q}}\right) .
$$

From Eqn. (64) it can be easily understood that if a face on a shell is divided into two by a curve on the face, the sum of singularities on the divided faces will be the same as that of the original face if the curve is one of the following two cases:

1. A smooth and closed curve. In this case, there are no extra vertex corners introduced and the sum of the $\chi$ does not change (e.g. Fig. 15 (b)).

2. A curve which intersects the boundary of the face and creates two oneelement-angles on each boundary. In this case, $\chi$ increases by 1 , and the second term on the right hand side of Eqn. (64) increases by 4 , therefore the sum does not change (e.g. Fig. 15 (c)).

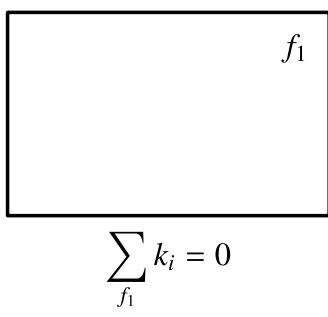

(a)

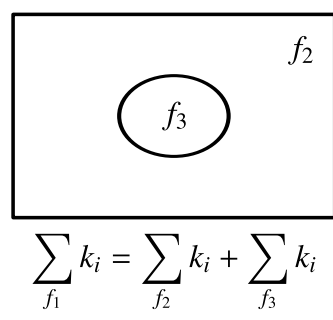

$=-4+4=0$

(b)

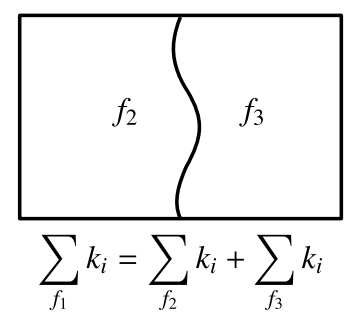

$=0+0=0$

(c)

Figure 15: (a) Original face with $\sum k_{i}=0$; (b) The difference $d$ of $\sum k_{i}$ compared to (a) is $d=-4(-1+1)+0=0$; (c) The difference $d$ of $\sum k_{i}$ compared to (a) is $d=-4(1)+4(2-1)=0$.

Therefore, if a face of the body is not homomorphic to a closed disc, it can always be divided using either of the above two kinds of curve, which will not change the sum of the singularities on the faces. As to the right-hand 
side of Eqn. (72), the above operations will not change $\chi$ of the shell of the 3 -D body. It will create some new vertices with $\sum_{q=1}^{\# F\left(v_{p}\right)} n_{c_{q p}}=4$, which means there is no need to count these new vertices. Therefore, Eqn. (72) holds in general cases. A few examples of calculating the sum of quad mesh singularity indices on the boundary faces using Eqn. (72) are given in Fig. 16.
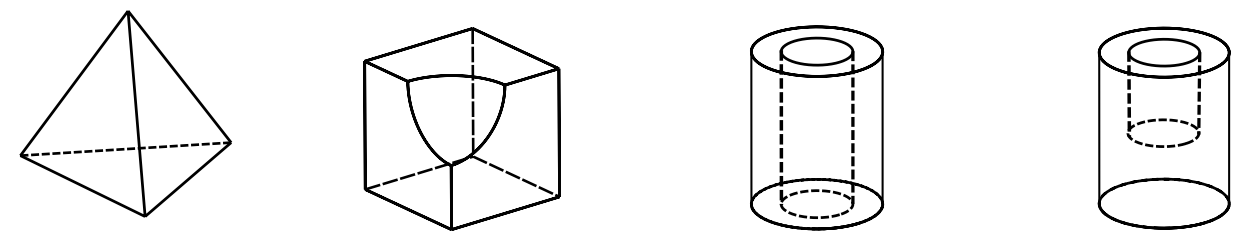

$$
\begin{aligned}
& \chi=2, \sum\left(4-\sum n_{\text {pal }}\right)=4 \quad \chi=2, \sum\left(4-\sum n_{\left.p^{*}\right)}=10\right. \\
& \Rightarrow \sum k_{i}=-4 \\
& \Rightarrow \sum k_{i}=+2
\end{aligned}
$$
$\chi=0, \sum\left(4-\sum n_{x}\right)=0$
$\Rightarrow \sum k_{i}=0$

Figure 16: Examples of calculating the sum of quad mesh singularities on the boundary faces of a shell of a 3-D body using Eqn. (72).

\subsection{Hex mesh singularities in a 3-D body}

\subsubsection{Definitions}

In a 3 -D hex mesh, let $V=\{v\}, E=\{e\}, H=\{h\}$ be the set of vertices, edges and hex elements respectively. In a grid or regular hex mesh, for an edge in the interior (not on the boundary), $e$,

$$
\# \operatorname{adj} H(e)=4
$$

where $\operatorname{adjH}(e)$ gives the set of adjacent hex elements to the edge. Let the line singularity index of an edge be

$$
k_{\text {line }}(e)=\# \operatorname{adj} H(e)-4
$$

and let the point singularity index of a vertex be

$$
k_{\text {point }}(v)=\sum_{i=0}^{\# \operatorname{adj} E(v)} k_{\text {line }}\left(e_{i}\right)
$$

where $a d j E(v)$ gives the set of adjacent edges to the vertex. For example in Fig. $17 k_{\text {line }}\left(e_{0}^{\ell}\right)=k_{\text {line }}\left(e_{1}^{\ell}\right)=1, k_{\text {point }}\left(v_{b}\right)=1$ and $k_{\text {point }}(v)=2$. Line 


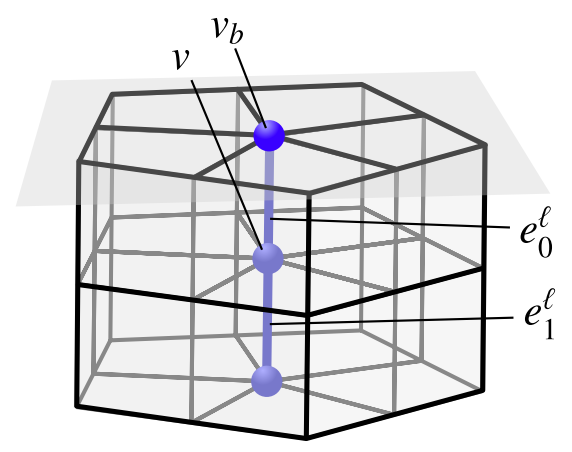

Figure 17: An example of a line singularity with index $c^{\ell}=1$ in a hex mesh.

singularities are defined as the connected series of edges, $E^{\ell}=\left(\ldots e_{i}^{\ell}, \ldots\right)$, with $k_{\text {line }}\left(e_{i}^{\ell}\right)=c^{\ell} \neq 0$ where $c^{\ell}$ is a non-zero integer that is constant for edges in $E^{\ell}$. For example, in Fig. $17, E^{\ell}=\left(e_{0}^{\ell}, e_{1}^{\ell}\right)$ and $c^{\ell}=1$. Let $\operatorname{adj} E^{\ell}(v)$ give the set of adjacent edges with $k_{\text {line }}(e) \neq 0$. So, in Fig. $17 \# a d j E^{\ell}\left(v_{b}\right)=1$ and $\operatorname{adj} E^{\ell}(v)=2$.

\subsubsection{Existing work}

The fundamental properties of hex meshes have been studied by Price et al. [14]. They demonstrate that line singularities can

- enter or exit the boundary of the $3-\mathrm{D}$ body at a vertex, $v_{b}$, with $k_{\text {point }}\left(v_{b}\right)=c^{\ell}$,

- form a closed loop in the interior of the body or

- combine with other line singularities.

The combination of line singularities is not arbitrary. They consider the convex polyhedrons formed by the hex elements sharing an internal vertex. If only simple line singularities with indices $\left|c^{\ell}\right|=1$ are considered then the polyhedrons have only 3, 4 and 5 sided faces. By starting with the Euler Formula and then simplifying using connectivity identities they derive the following identity

$$
3 F_{3}+2 F_{4}+F_{5}=12
$$

where $F_{3}, F_{4}$ and $F_{5}$ are the numbers of 3-, 4- and 5-sided polygon faces of the polyhedron. The 12 possible polyhedrons that satisfy this equation are enumerated and termed the 'hex mesh primitives'. It is suggested that 
singularities of higher order $\left(\left|c^{\ell}\right|>1\right)$ correspond to the superposition of these hex mesh primitives.

Nieser et al. [15] derived an equivalent condition to Eqn. (76) for the possible combinations of line singularities. In their approach a small sphere surrounding an internal vertex, $v_{c}$, is considered. The hex elements connected

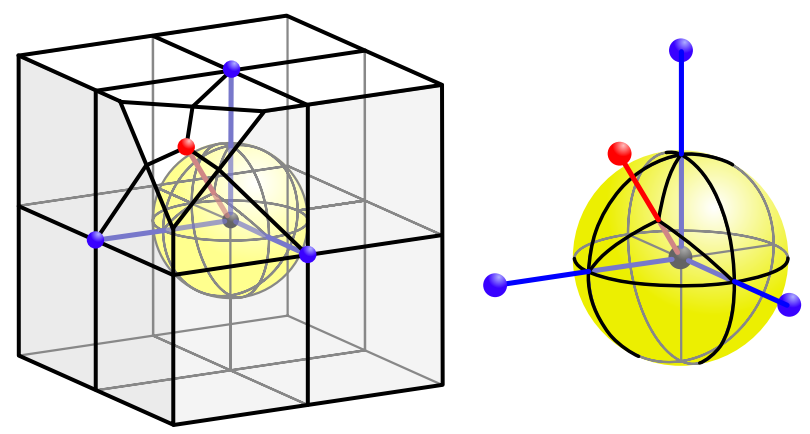

Figure 18: A small sphere around an internal vertex of a hex mesh with an induced tri mesh.

to the internal vertex induce a tri mesh on the surface of the sphere, as shown in Fig. 18. In a tri mesh with vertices, edges and tris $V, E$ and $F$,

$$
\sum_{i=0}^{\# V} \text { valence }\left(v_{i}\right)=3 \# F .
$$

Using Eqn. 54 in Sec. 5.2,

$$
2 \# V-\# E_{b}+(2-P) \# F=2 \chi
$$

and substituting $\# E_{b}=0, P=3, \chi=2$ gives

$$
2 \# V-\# F=4 \text {. }
$$

Combining Eqns. (77) and (79) gives

$$
\sum_{i=0}^{\# V}\left(6-\operatorname{valence}\left(v_{i}\right)\right)=12 .
$$

Since the valence of a vertex in the tri mesh on the sphere corresponds to the number of adjacent hex elements of the edge that travels through the sphere 
at that vertex, Eqn. (80) can be rewritten in terms of the edges connected to $v_{c}, \operatorname{adj} E\left(v_{c}\right)$, as

$$
\sum_{i=0}^{\# \operatorname{adj} E\left(v_{c}\right)}\left(6-\# \operatorname{adj} H\left(e_{i}\right)\right)=\sum_{i=0}^{\# \operatorname{adj} E\left(v_{c}\right)}\left(2-k_{\text {line }}\left(e_{i}\right)\right)=12 .
$$

\subsubsection{Formula relating line and point singularities in a hex mesh}

Critical vertices in a hex mesh can be defined as boundary vertices connected to line singularities and junction vertices in the interior where there are 3 or more line singularities combine. Let $V^{*}$ be the set of all critical vertices with independent subsets $V_{b}^{*}$ and $V_{J}^{*}$ for the critical boundary and junction vertices,

$$
\begin{aligned}
& V^{*}=\left\{\operatorname{adj} E^{\ell}(v) \neq 0 \mid v \in V\right\}, \\
& V_{b}^{*}=\left\{\operatorname{adj} E^{\ell}(v)=1 \mid v \in V\right\}, \\
& V_{J}^{*}=\left\{\operatorname{adj} E^{\ell}(v) \geq 3 \mid v \in V\right\} .
\end{aligned}
$$

$V_{b}^{*}$ and $V_{J}^{*}$ can be subdivided into independent subsets

$$
\begin{array}{c|c}
V_{b}^{*}=\left.\left.V_{b}^{*}\right|_{b-b} \cup V_{b}^{*}\right|_{b-J} & \left.\left.V_{b}^{*}\right|_{b-b} \cap V_{b}^{*}\right|_{b-J}=\emptyset, \\
V_{J}^{*}=\left.\left.V_{b}^{*}\right|_{J-J} \cup V_{b}^{*}\right|_{J-b} & \left.\left.V_{b}^{*}\right|_{J-J} \cap V_{b}^{*}\right|_{J-b}=\emptyset .
\end{array}
$$

The subscript left-right notation stands for: left - the type of critical vertex, $b$ for boundary or $J$ for junction, right: where the line singularity connected to it terminates, $b$ for boundary or $J$ for junction. From the definitions of the subsets $\left.\# V_{b}^{*}\right|_{b-J}=\left.\# V_{b}^{*}\right|_{J-b}$.

By the definitions in Eqns. (74), (75) and (82)

$$
\sum_{i=0}^{\# V^{*}}\left(k_{\text {point }}\left(v_{i}\right)-\sum_{j=0}^{\# a d j E^{\ell}\left(v_{i}\right)} k_{\text {line }}\left(e_{j}\right)\right)=0
$$

and separating $\# V^{*}$ out gives

$$
\begin{aligned}
& \sum_{i=0}^{\# V_{b}^{*}} k_{\text {point }}\left(v_{i}\right)+\sum_{j=0}^{\# V_{J}^{*}} k_{\text {point }}\left(v_{j}\right) \\
& -2 \sum_{k=0}^{\# V_{b-J}^{*} \# a d j E^{\ell}\left(v_{k}\right)} \sum_{l=0} k_{\text {line }}\left(e_{l}\right)-\sum_{m=0}^{\# V_{b-b}^{*} \# a d j E^{\ell}\left(v_{m}\right)} \sum_{n=0} k_{\text {line }}\left(e_{n}\right)-\sum_{o=0}^{\# V_{J-J}^{*} \# a d j E^{\ell}\left(v_{o}\right)} k_{p=0} k_{\text {line }}\left(e_{p}\right)=0 .
\end{aligned}
$$


This formula is another condition, along with Eqn. (81), that applies to networks of line singularities for them to permit valid corresponding hex meshes.

The critical boundary singularities of the mesh can be divided into two separate subsets

$$
V_{b}^{*}=\left.\left.\left.V_{b}^{*}\right|_{\text {vertex }} \cup V_{b}^{*}\right|_{\text {face }}\left|V_{b}^{*}\right|_{\text {vertex }} \cap V_{b}^{*}\right|_{\text {face }}=\emptyset,
$$

where $\left.V_{b}^{*}\right|_{\text {vertex }}$ contains critical vertices at vertices of the body and $\left.V_{b}^{*}\right|_{\text {face }}$ contains critical vertices on faces of the body. Similarly to vertex classification in 2-D (Fig. 3), vertex classification in 3-D involves selecting the numbers of hex elements to occur at a vertex of the body, and thereby specifying the point singularity index of the vertex of the mesh. Examples of two possible classifications of a vertex of a body connected to 3 faces and 3 edges are shown in Fig 19. The selection is not unconstrained however and
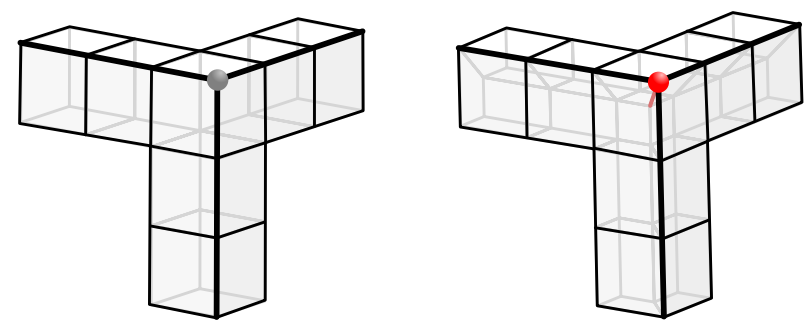

Figure 19: Examples of vertex classifications in 3-D.

the vertex classifications for the end vertices of an edge must be compatible. After selecting the vertex classifications for the body's vertices the value of $\sum_{i=0}^{\left.V_{b}^{*}\right|_{\text {vertex }}} k_{\text {point }}\left(v_{i}\right)$ is set. Also, the value of $\sum_{i=0}^{\left.V_{b}^{*}\right|_{\text {face }}} k_{\text {point }}\left(v_{i}\right)$ is set since from Eqn. (72),

$$
\left.V_{b}^{*}\right|_{\text {face }}=\sum_{i=0}^{\left.\# P_{k}\right|_{\text {shell }}} k_{i}=-4 \chi(\text { shell })+\sum_{p=1}^{\left.\# V\right|_{\text {shell }}}\left(4-\sum_{q=1}^{\left.\# F\left(v_{p}\right)\right|_{\text {shell }}} n_{c_{p q}}\right),
$$

and the vertex classifications gives $n_{c}$ for the attached faces. So, the first term on the LHS of Eqn. (88) is set by the vertex classifications and the topologies of the body's shells. The rest of the terms on the LHS are variable with many possibilities but a network of line singularities of any valid hex mesh of the 3-D body must be such that they give equality. 


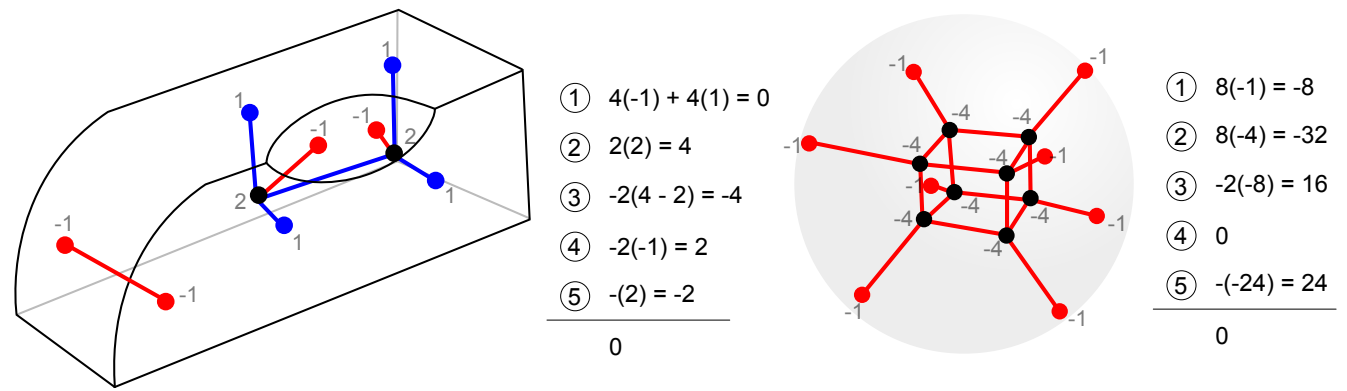

Figure 20: Two examples of validating simple line singularity networks.

Two examples of validating simple line singularity networks in bodies using Eqn. (88) are shown in Fig. 20. The point singularity indices of the critical vertices are marked. The LHS terms 1-5 in Eqn. (88) are listed and shown to sum to zero.

\section{Potential applications}

\subsection{Identifying sweepable volumes}

Sweeping is one of the most popular approaches to hex mesh generation. It takes the quad meshes generated on the source faces and extrudes them along a specified direction to the target faces. There are three types of sweeping meshing algorithms: one-to-one, one-to-many and many-to-many, each depending on the number of source and target faces. Any body which can be meshed using a sweeping algorithm is referred to as sweepable volume. It is straightforward to understand that the wall faces of a sweepable volume should be structured with zero singularities, as shown in Fig. 21.

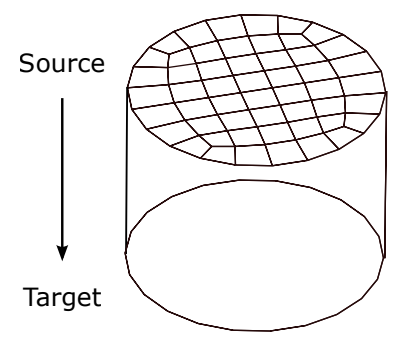

(a)

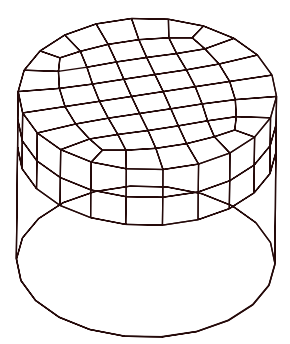

(b)

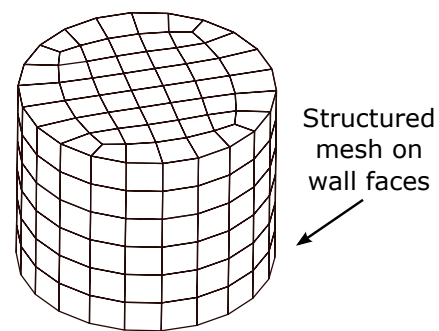

(c)

Figure 21: Example of the one-to-one sweeping. 
White et al. [2] have developed an algorithm to identify sweepable volume based on the classification of vertex and edge types. They use a version of Eqn. (1) to identify submappable faces. Eqn. (22) is an equally simple but more descriptive meshing validity check and can be used for this purpose and more. Fig. 22 shows a few examples of identified sweepable volumes using a similar algorithm to [2] that uses Eqn. (22). The sweeping paths of the
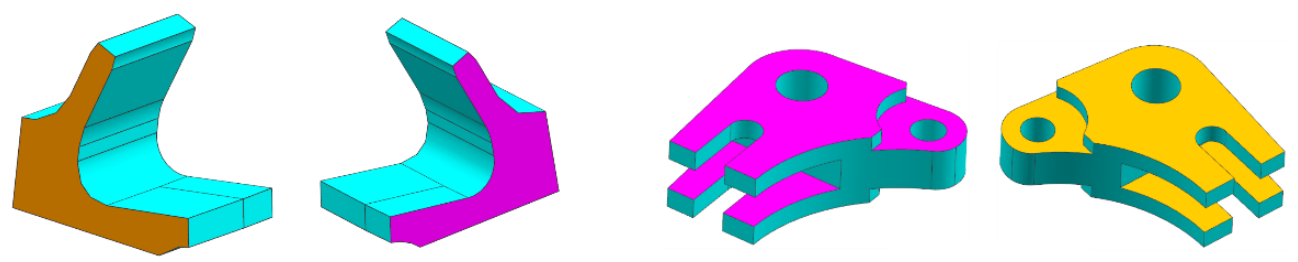

Figure 22: Example of identified sweepable volumes

above mentioned sweepable volumes are all open curves. An extension of using Eqn. (22) as opposed to Eqn. (1) is that a volume can be identified as being suitable for sweep meshing, where the mesh is formed by sweeping a surface mesh along a smooth closed curve (including those formed by revolving around an axis). A feature of this type of sweepable volume is that each face has zero mesh singularities with $\chi=0$ and $\sum\left(2-n_{c_{i}}\right)=0$ in Eqn. (22). This differs from a submappable volume, where each face also has zero mesh singularities, but with $\chi=1$ and $\sum\left(2-n_{c_{i}}\right)=4$, as shown in Fig 23.

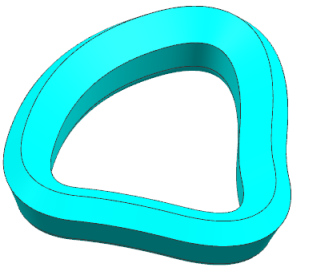

(a)

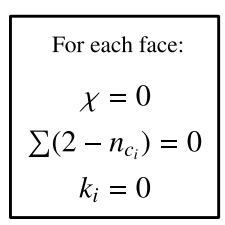

.

Figure 23: (a) sweepable volume where the sweeping path is a smooth and closed curve; (b) submappable volume.

\subsection{Locating mesh singularities}

Equation (22) shows that the net sum of the singularities is determined by the Euler Characteristic, $\chi$, and the number of elements at each corner. 
Suppose a small offset is made to the boundaries of a surface as shown in Fig. 24. If the number of mesh singularities does not change for the surface

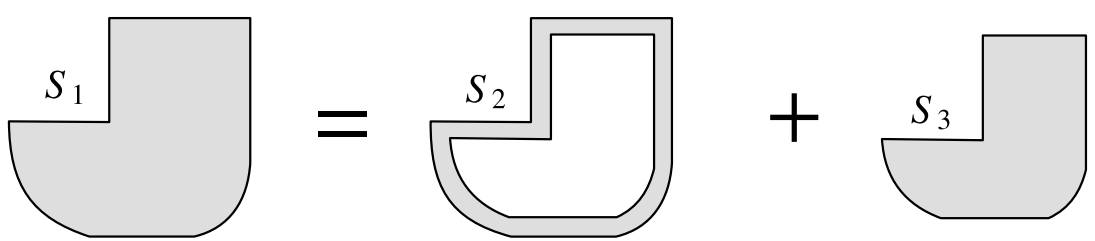

Figure 24: Making an offset of the boundaries of the original surface.

after offset $S_{3}$, then it can be claimed the net number of mesh singularities in the offset surface $S_{2}$ is zero ( $S_{2}$ has no mesh singularities or pairs of positive and negative singularities). If the number of mesh singularities in $S_{1}$ and $S_{3}$ is different, a mesh singularity should appear in $S_{2}$. A reasonable place to locate the singularity is in the region of the corner where the number of elements or the Euler Characteristic changes. The offset operation can be made until the shape after offset is small and the number of mesh singularities stops varying. Therefore, by making the offset of the boundaries inwards and tracing the change of the element number or Euler Characteristic at each corner, the singularities can be located.

Fig. 25 (a) shows a squeezed rectangle with a net number of zero mesh singularities. The net number changes from 0 to 1 when offsetting from the red contour to the blue contour. This is caused by the angle of the rightmost corner passing though $45^{\circ}$ and its vertex type switching from $n_{c}=0$ to $n_{c}=1$. Therefore, a positive mesh singularity can be placed between the blue and red lines in the region of the right corner. With further offsets, the shape becomes a triangle and the element number does not change any more. For a triangle with one element at each corner, one negative singularity is required, as the red point shows. Fig. 25 (b) shows an example where the Euler Characteristic changes during the offset process. There is one positive singularity for the original shape. When it offsets to the position as shown the original shape becomes two separate shapes with zero mesh singularities. Therefore the positive singularity will be placed at the transition position. In principle, the offset distance needs to be small. But it is still possible that a pair of positive and negative singularities exist within one offset, as shown in Fig. 25 (c). However, by checking whether the number of elements or the Euler Characteristic changes at each corner of the offset geometry, the positive and negative singularities can still be properly located. Examples 

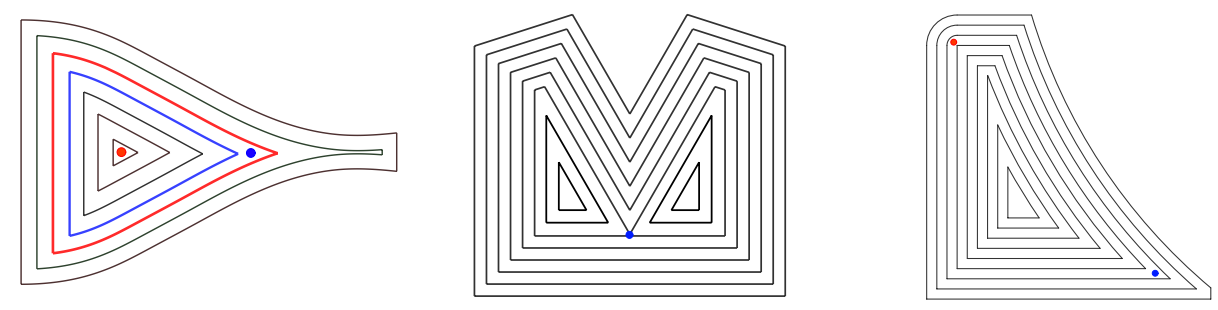

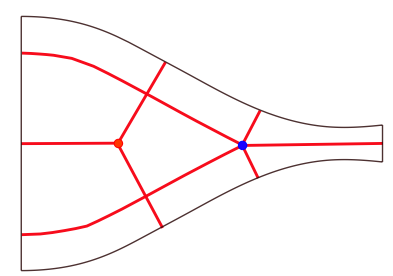

(a)

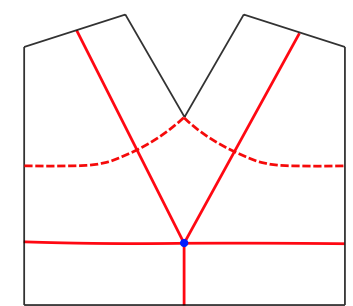

(b)

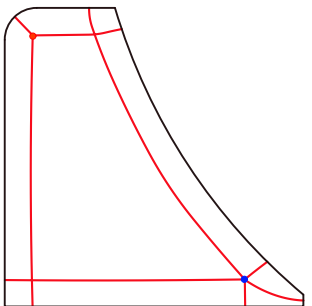

(c)

Figure 25: Examples of locating mesh singularities using offsets: (a) the element number at the corner changes; (b) the Euler Characteristic changes; (c) a pair of positive and negative mesh singularities within one offset.

of the manual block decomposition are given in the second row in Fig. 25. In fact, the offset boundaries offer some clues for the decomposition lines, e.g. some lines follow the offset boundaries and some others are roughly perpendicular to them. The construction of these partitioning lines is not the focus here and will not be discussed in detail, but is an interesting avenue for further research.

A more complex example is given in Fig. 26 (a) where an analogy between the MAT (Medial Axis Transformation) and offsetting methods is drawn. In the offsetting method here, a singularity is identified when the number of element at the corner or Euler Characteristics changes and the singularity is placed near the corner. Fig. 26 (b) shows the result of the identified singularities using the MAT method in [16], where the domain is partitioned into tracks with two radii running from the medial axis perpendicular to the boundary. A flux balance analysis is carried out for each pair of medial radii. The flux difference between two pairs of medial radii comprising a track indicates the existence and type of mesh singularities, which are placed on the medial axis. It can be seen that the results given by both approaches are similar for this body.

With the offsetting boundary method it is also possible to locate the line 


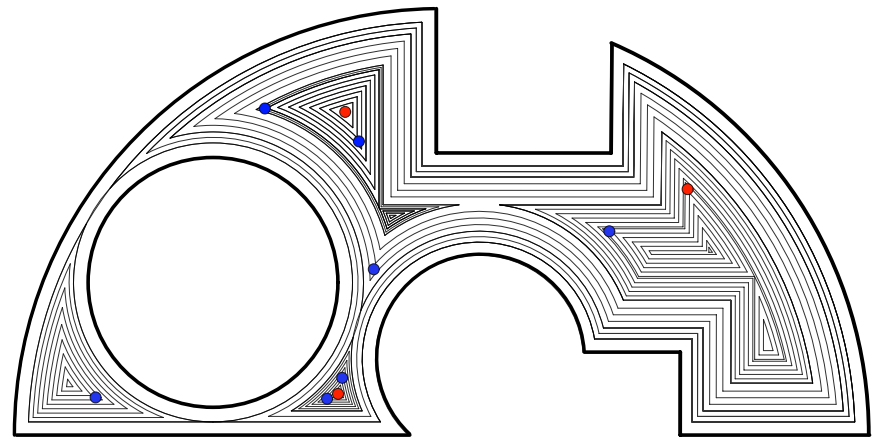

(a)

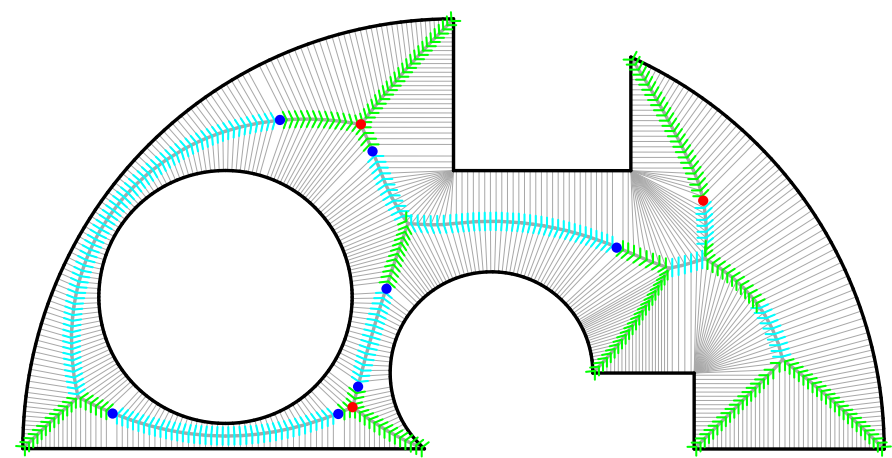

(b)

Figure 26: Mesh singularities calculated by making offsets of the boundary (a) and by MAT method (b) (Taken and adapted from [16])

singularities in 3-D for hex meshing. Similarly, the internal singularity points can be placed at corners where the term $\sum_{q=1}^{\# F\left(v_{p}\right)} n_{c_{p q}}$ or Euler Characteristic in Eqn. (72) changes. The body in Fig. 27 is a cube with three equal blends, Fig. 27 (a). Fig. 27 (b) shows the result after the first offset. It can be easily understood that there will be no singularities in the region between Fig. 27 (a) and Fig. 27 (b). However, after the second offset, Fig. 27 (c), $\sum_{q=1}^{\# F\left(v_{p}\right)} n_{c_{p q}}$ changes at four corners which indicates a point singularity. The common edges between these corners indicate that these internal singularity points should be joined together. Each internal singularity points can then be connected to the surface singularity point. Further research is necessary to formulate the detailed rules for the placement of line singularities following this approach. 


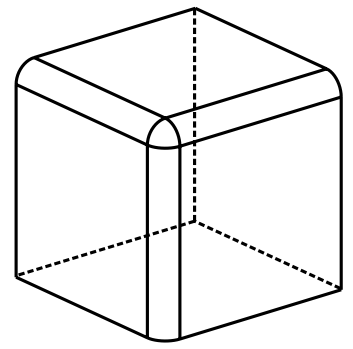

(a)

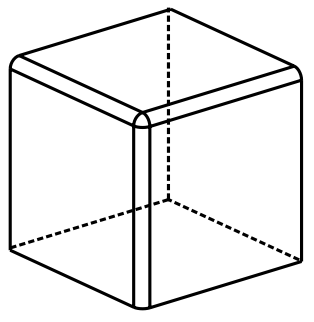

(b)

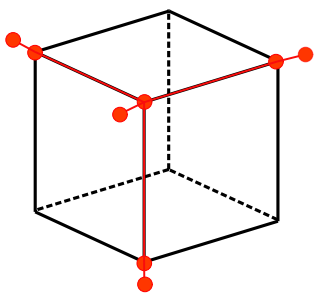

(c)

Figure 27: A 3-D example of locating line singularities by offsetting the boundaries.

\section{Conclusion}

A proof of a simple formula for the possible combinations of singularities in a boundary aligned cross-field and boundary conforming quad and tri mesh on a face has been presented. The only parameters involved are the vertex classification types and the Euler Characteristic of the face. Its sufficiency has also been discussed. The use of the formula has been demonstrated on a range of faces to provide an intuitive validation of its correctness. For 3-D bodies a new condition to verify line singularity networks for hex meshes has been presented. Some potential applications including the identification of sweepable volumes and locating quad and hex mesh singularities by an offsetting approach were outlined.

\section{Acknowledgements}

The authors from the Meshing and Abstraction Group, Simcenter, Siemens PLM would like to thank their manager Mary Otte for her support and encouragement in doing this work. The authors from Queen's University Belfast would like to acknowledge the financial support provided by Innovate UK, a UK Centre for Aerodynamics project, via GHandI project (TSB 101372). The QUB authors also acknowledge Rolls-Royce for granting permission to publish this paper.

\section{References}

[1] G. Bunin, A continuum theory for unstructured mesh generation in two dimensions, Comput. Aided Geom. Des. 25 (1) (2008) 14-40. 
[2] D. R. White, T. J. Tautges, Automatic scheme selection for toolkit hex meshing, International Journal for Numerical Methods in Engineering 49 (1-2) (2000) 127-144. doi:10.1002/1097-0207(20000910/20)49: $1 / 2<127$ : : AID-NME926>3. 0. CO ; 2-V.

[3] E. Ruiz-Girones, J. Sarrate, Generation of structured meshes in multiply connected surfaces using submapping, Advances in Engineering Software 41 (2) (2010) 379 - 387. doi:10.1016/j .advengsoft.2009.06.009.

[4] P.-A. Beaufort, J. Lambrechts, F. Henrotte, C. Geuzaine, J.-F. Remacle, Computing cross fields A PDE approach based on the Ginzburg-Landau theory, Procedia Engineering 203 (2017) 219 - 231, 26th International Meshing Roundtable, IMR26, 18-21 September 2017, Barcelona, Spain. doi:https://doi.org/10.1016/j.proeng.2017.09.799.

[5] D. Bommes, B. Lévy, N. Pietroni, E. Puppo, C. S. a, M. Tarini, D. Zorin, State of the art in quad meshing, in: Eurographics STARS, 2012.

[6] A. Vaxman, M. Campen, O. Diamanti, D. Bommes, K. Hildebrandt, M. Ben-Chen, D. Panozzo, Directional field synthesis, design, and processing, in: SIGGRAPH ASIA 2016 Courses, SA '16, ACM, New York, NY, USA, 2016, pp. 15:1-15:30. doi:10.1145/2988458.2988478.

[7] J. Palacios, E. Zhang, Rotational symmetry field design on surfaces, ACM Trans. Graph. 26 (3) (2007) 55. doi:10.1145/1276377.1276446.

[8] N. Ray, B. Vallet, W. C. Li, B. Lévy, N-symmetry direction field design, ACM Trans. Graph. 27 (2) (2008) 10:1-10:13. doi:10.1145/1356682. 1356683.

[9] F. Knöppel, K. Crane, U. Pinkall, P. Schröder, Globally optimal direction fields, ACM Trans. Graph. 32 (4) (2013) 59:1-59:10. doi: 10.1145/2461912.2462005.

[10] M. P. do Carmo, Differential Geometry of Curves and Surfaces, 1st Edition, Pearson, 1976.

[11] O. Diamanti, A. Vaxman, D. Panozzo, O. Sorkine-Hornung, Designing n-polyvector fields with complex polynomials, Comput. Graph. Forum 33 (5) (2014) 1-11. doi:10.1111/cgf.12426. 
[12] Siemens Simcenter 3D, www.plm.automation.siemens.com/en_us/ products/simcenter/3d/modeling/index.shtml, (26-May-2017).

[13] J. Jackson, Classical electrodynamics, Wiley, New York, 1999.

[14] M. A. Price, C. G. Armstrong, M. A. Sabin, Hexahedral mesh generation by medial surface subdivision: Part I. Solids with convex edges, International Journal for Numerical Methods in Engineering 38 (19) (1995) 3335-3359. doi:10.1002/nme.1620381910.

[15] M. Nieser, U. Reitebuch, K. Polthier, Cubecover parameterization of 3D volumes, Computer Graphics Forum 30 (5) (2011) 1397-1406. doi : 10.1111/j.1467-8659.2011.02014.x.

[16] H. J. Fogg, C. G. Armstrong, T. T. Robinson, Enhanced medialaxis-based block-structured meshing in 2-d, Computer-Aided Design 72 (2016) 87 - 101, 23rd International Meshing Roundtable Special Issue: Advances in Mesh Generation. doi:10.1016/j.cad.2015.07.001. 